\title{
Subcellular Localization and Activity of the Mitogen-Activated Protein Kinase Kinase 7 (MKK7) $\gamma$ Isoform are Regulated through Binding to the Phosphatase Calcineurin
}

\author{
Emily S. Gibson, Kevin M. Woolfrey, Huiming Li, ${ }^{1}$ Patrick G. Hogan, Raphael A. Nemenoff, \\ Lynn E. Heasley, and Mark L. Dell'Acqua
}

Department of Pharmacology (E.S.G., K.M.W., M.L.D.) and Department of Medicine, Division of Renal Diseases and Hypertension (R.A.N.), University of Colorado School of Medicine, Aurora, Colorado; Department of Craniofacial Biology, University of Colorado School of Dental Medicine, Aurora, Colorado (L.E.H.); Immune Disease Institute, Harvard Medical School, Boston, Massachusetts (H.L.); and La Jolla Institute for Allergy and Immunology, La Jolla, California (P.G.H.)

Received May 24, 2018; accepted October 31, 2018

\section{ABSTRACT}

Calcineurin $(\mathrm{CaN})$ phosphatase signaling is regulated by targeting $\mathrm{CaN}$ to substrates, inhibitors, and scaffold proteins containing docking motifs with the consensus sequence of PxIxIT. Here, we identify the docking of CaN to the $\gamma$ isoform of MKK7, a component of the c-Jun N-terminal kinase (JNK) pathway. Because of alternative splicing of a single exon within the $\mathrm{N}$-terminal domain, MKK7 $\gamma$ encodes a unique PxIxIT motif (PIIVIT) that is not present in $\mathrm{MKK} 7 \alpha$ or $\beta$. We found that MKK7 $\gamma$ bound directly to CaN through this PIIVIT motif in vitro, immunoprecipitated with CaN from cell extracts, and exhibited fluorescence resonance energy transfer (FRET) with $\mathrm{CaN}$ in the cytoplasm but not in the nucleus of living cells. In contrast, MKK $7 \alpha$ and $\beta$ exhibited no direct binding or FRET with $\mathrm{CaN}$ and were localized more in the nucleus than the cytoplasm. Furthermore, the inhibition of $\mathrm{CaN}$ phosphatase activity increased the basal phosphorylation of MKK7 $\gamma$ but not MKK7 $\beta$. Deletion of the MKK7 $\gamma$ PIIVIT motif eliminated FRET with CaN and promoted $\mathrm{MKK} 7 \gamma$ redistribution to the nucleus; however, the inhibition of $\mathrm{CaN}$ activity did not alter $\mathrm{MKK} 7 \gamma$ localization, indicating that MKK7 $\gamma$ cytoplasmic retention by $\mathrm{CaN}$ is phosphatase activity independent. Finally, the inhibition of CaN phosphatase activity in vascular smooth muscle cells, which express MKK7 $\gamma$ mRNA, enhances JNK activation. Overall, we conclude that the MKK7 $\gamma$-specific PxIxIT motif promotes high-affinity CaN binding that could promote novel cross talk between $\mathrm{CaN}$ and JNK signaling by limiting MKK7 $\gamma$ phosphorylation and restricting its localization to the cytoplasm.

\section{Introduction}

The ubiquitous phospho-ser/thr protein phosphatase calcineurin (CaN; also known as PP2B and PPP3) controls many important cellular functions, including the immune response in $\mathrm{T}$ cells, hypertrophic growth of myocytes, and synaptic plasticity in neurons. Elevations in intracellular $\mathrm{Ca}^{2+}$ stimulate the phosphatase activity of the CaN A catalytic subunit

This research was supported by grants from the National Institutes of Health (NIH) to M.L.D. [MH-102338 and NS-040701], to Anjana Rao (La Jolla Institute for Allergy and Immunology, La Jolla, CA) and P.G.H. [AI-040127], to H.L. [AI-090428], to L.E.H. [CA-116527], and to R.A.N. [CA-162226 and CA-058187]. The contents of this article are the authors' sole responsibility and do not necessarily represent official NIH views. No potential conflicts of interest relevant to this article are reported.

${ }^{1}$ Current affiliation: Acceleron Pharma, Cambridge, Massachusetts.

https://doi.org/10.1124/mol.118.113159. through $\mathrm{Ca}^{2+}$ binding to a preassociated CaN B regulatory subunit and to calmodulin, which binds adjacent to CaNB subunit on the CaN A subunit C-terminal regulatory domain. The concerted actions of $\mathrm{Ca}^{2+}-\mathrm{CaNB}$ and $\mathrm{Ca}^{2+}-\mathrm{CaM}$ then cause a conformational change in the CaNA regulatory domain that leads to displacement of an autoinhibitory domain from the catalytic active site (Li et al., 2011). However, the CaNA subunit also engages in additional protein-protein interactions with a variety of binding partners that contain docking motifs with the consensus sequence of PxIxIT. These diverse CaN-PxIxIT docking interactions can in turn regulate the specificity of $\mathrm{CaN}$ signaling by controlling substrate recognition, catalytic activity, and subcellular targeting to multiprotein signaling complexes by scaffold proteins (Aramburu et al., 1998; Oliveria et al., 2007; Li et al., 2011;

ABBREVIATIONS: 3F, three filter; AKAP79, A-kinase anchoring protein 79; AVP, arginine-vasopressin; CaN, calcineurin; CFP, cyan fluorescent protein; CFPpost, cyan fluorescent protein donor intensity after; CFPpre, cyan fluorescent protein donor intensity before; CsA, cyclosporine A; DTT, dithiothreitol; Eeff, fluorescence resonance energy transfer efficiency; FRET, fluorescence resonance energy transfer; FRETc, corrected, sensitized fluorescence resonance energy transfer; GAPDH, glyceraldehyde-3-phosphate dehydrogenase; GFP, green fluorescent protein; GST, glutathione S-transferase; HEK, human embryonic kidney; IP, immunoprecipitation; JNK, c-Jun N-terminal kinase; MAPK, mitogen-activated protein kinase; MAPKKK, mitogen-activated protein kinase kinase kinase; MKK7, mitogen-activated protein kinase kinase 7; NFAT, nuclear factor of activated T cells; PB, photobleaching; PBS, phosphate-buffered saline; TLB, Triton Lysis Buffer; PCR, polymerase chain reaction; PKA, cAMP-dependent protein kinase A; qRT-PCR, quantitative real-time polymerase chain reaction; rVSMC, rat vascular smooth muscle cell; VSMC, vascular smooth muscle cell; YFP, yellow fluorescent protein. 
Sanderson et al., 2012; Grigoriu et al., 2013; Murphy et al., 2014).

The PxIxIT docking motif was originally identified in studies that characterized the mechanism by which CaN first recognizes and then dephosphorylates the nuclear factor of activated $\mathrm{T}$ cells (NFAT) family of transcription factors to promote NFAT translocation from the cytoplasm to the nucleus to control gene expression (Aramburu et al., 1998). Subsequent peptide library screening identified the PVIVIT peptide as an artificial PxIxIT motif with submicromolar CaN binding affinity (Aramburu et al., 1999). X-ray crystallographic determination of the structure of the PVIVIT-CaN complex elucidated the molecular basis CaN A subunit binding to PxIxIT motifs through a $\beta$-sheet interaction at a site distant from the phosphatase catalytic site, thus explaining how PxIxIT binding can direct either active or inactive $\mathrm{CaN}$ to specific substrates (Li et al., 2007). Interestingly, there is considerable nonconsensus variation in the PxIxIT motif sequences for different $\mathrm{CaN}$ binding proteins, leading to a wide range of binding affinities, with $\mathrm{K}_{\mathrm{d}}$ (dissociation constant) values from submicromolar to hundreds of micromolar (Li et al., 2011; Goldman et al., 2014). For instance, our recent $\mathrm{X}$-ray structure-function analysis of the postsynaptic plasma membrane scaffold protein A-kinase anchoring protein 79 (AKAP79) identified an IAIIIT variant of this motif that interacts with $\mathrm{CaN}$ at the same site as PVIVIT but with Ile substituting for Pro in the first position ( $\mathrm{Li}$ et al., 2012). However, this AKAP79 nonconsensus variant motif binds $\mathrm{CaN}$ with submicromolar affinity, which is similar to PVIVIT and actually is greater than all other known naturally occurring consensus PxIxIT sequences.

An important feature of AKAP79 is that it not only binds $\mathrm{CaN}$ to target it to specific substrates but also anchors two opposing protein kinases, the cAMP-dependent protein kinase $\mathrm{A}$ (PKA) and the $\mathrm{Ca}^{2+}$ and phospholipid-activated protein kinase $\mathrm{C}$, thus allowing for coordinated bidirectional regulation of phosphorylation and integration of different second messenger pathways within a single signaling complex (Woolfrey and Dell'Acqua, 2015; Wild and Dell'Acqua, 2018). Based on the AKAP79-CaN/PKA/protein kinase C scaffold model, we sought to identify other signaling proteins that could anchor $\mathrm{CaN}$ and protein kinases in the same signaling complex. To this end, we used a combination of in silico, biochemical, and cellular imaging approaches to identify and characterize a consensus PxIxIT CaN docking motif in the $\gamma$ isoform splice variant of the mitogen-activated protein kinase (MAPK) kinase 7 (MKK7; also known as MAPKK7), a key upstream kinase regulator of JNK signaling. In particular, we demonstrate that this PxIxIT motif promotes high-affinity CaN binding to MKK7 $\gamma$, selectively regulates MKK7 $\gamma$ phosphorylation, and restricts localization of $M K K 7 \gamma$ to the cytoplasm instead of the nucleus, where the related $\mathrm{MKK} 7 \alpha$ and $\beta$ isoforms lacking this motif are concentrated. Importantly, we also provide evidence that this scaffolding of CaN to MKK7 $\gamma$ may promote cross talk between CaN and JNK signaling in vascular smooth cells.

\section{Materials and Methods}

cDNA Expression Plasmids and Transfection of Human Embryonic Kidney-293 and COS7 Cells. Previous publications describe plasmids encoding $\mathrm{CaNA} \alpha$-myc (Dell'Acqua et al., 2002);
CaNA $\alpha$-CFP/YFP (Oliveria et al., 2003); CaNA $\alpha$ H151A-YFP (Oliveria et al., 2012); PVIVIT-GFP (Aramburu et al., 1999); and Flag-MKK $7 \alpha 1$, $\beta 1$, and $\gamma 1$ isoforms (Tournier et al., 1999). N-terminally tagged YFP and CFP versions of MKK $7 \alpha 1, \beta 1$, and $\gamma 1$ were constructed by transfer of Flag-MKK7 encoding HindIII-XbaI fragments into pEC/YFPC1 (Takara Bio USA, Inc., Mountain View, CA). The $\Delta$ PIX mutation deleting residues 41PIIVIT46 in MKK7 $\gamma 1$ was introduced by Stratagene QuikChange polymerase chain reaction (PCR) (Agilent Technologies Inc., Santa Clara). Human embryonic kidney (HEK)-293 and COS7 cells at $20 \%-50 \%$ confluency (24-48 hours after plating) were transfected by calcium phosphate precipitation with the various plasmid cDNA expression constructs [1-2 $\mu \mathrm{g}$ each plasmid per 25-mm glass coverslip in six-well plates for fluorescence resonance energy transfer (FRET) imaging or 2-10 $\mu \mathrm{g}$ each plasmid for $10-\mathrm{cm}$ plates for immunoprecipitation (IP)] for 4-6 hours at $5 \% \mathrm{CO}_{2}$ and $37^{\circ} \mathrm{C}$. Cells were then washed with phosphate-buffered saline (PBS), fed with normal growth medium (Dulbecco's modified Eagle's medium, $10 \%$ fetal bovine serum, $1 \%$ penicillin/streptomycin; Invitrogen, Carlsbad, CA) and grown for 24-48 hours prior to fluorescence imaging or preparation of cell extracts for IP.

IP of CaN-MKK7 Complexes from HEK-293 Cell Extracts. We analyzed Flag-MKK7 and CaNA-myc binding by co-IP from transfected HEK-293 cell extracts essentially as described for AKAP79 binding to CaNA-myc (Dell'Acqua et al., 2002). In Fig. 8, cells were treated with $1 \mu \mathrm{M}$ ionomycin (Tocris Bioscience, Bristol, UK) for 3 minutes prior to co-IP. Briefly, HEK-293 cells on 10-cm dishes were washed twice in PBS prior to harvesting. Cell extracts were prepared by lysis in ice-cold buffer containing $0.5 \%$ Triton X-100 [Triton Lysis Buffer (TLB): 20 mM Hepes, pH 7.4; 100 mM $\mathrm{NaCl} ; 5 \mathrm{mM}$ EDTA, $0.5 \%$ Triton X-100 (w/v), $1 \mathrm{mM}$ dithiothreitol (DTT); $2 \mathrm{mg} / \mathrm{ml}$ leupeptin/pepstatin; $1 \mathrm{mM}$ benzamidine; $1 \mathrm{mM}$ AEBSF (4-(2-aminoethyl) benzenesulfonyl fluoride)] followed by clarification of the lysate with centrifugation at $16,000 \mathrm{~g}$ for 15 minutes. Approximately $1.0 \mathrm{mg}$ of cell lysate was incubated at $4^{\circ} \mathrm{C}$ overnight with $2 \mu \mathrm{g}$ of mouse anti-myc (9E10; Santa Cruz Biotechnology, Dallas, TX), rabbit anti-Flag (sc-807; Santa Cruz Biotechnology), or unrelated mouse or rabbit nonimmune IgG. After antibody incubations, Pierce Protein A-Sepharose (Thermo Fisher Scientific, Waltham, MA) (25 $\mu \mathrm{l}$, $50 \%$ slurry equilibrated in TLB) was added and incubated for 1 hour at $4^{\circ} \mathrm{C}$. The immunecomplexes were pelleted by microcentrifugation (3000g, 1 minute), and the beads were washed in TLB $(4 \times 1 \mathrm{ml})$. The immunecomplexes were eluted with SDS-PAGE sample buffer, separated by SDS-PAGE and transferred to nitrocellulose filters. Filters were analyzed by immunoblotting with anti-Flag (1:1000), anti-myc (1:1000), or rabbit anti-GFP (1:500; Invitrogen) and horseradish peroxidase-coupled secondary antibodies (Bio-Rad, Hercules, $\mathrm{CA})$. Immunoblots were then visualized using enhanced chemiluminescence (Thermo Fisher Scientific) and a FluorChem gel documentation system (Alpha Innotech, San Leandro, CA).

IP of MKK7 species for phosphorylation analysis was performed as previously described (Woolfrey et al., 2015) with modifications as detailed below: HEK-293FT cells were transfected with myc-CaN, and YFP-MKK7 $\beta 1$ or YFP-MKK7 $\gamma 1$ were transfected by calcium phosphate precipitation, as described above. Cells were then treated with CaN inhibitors cyclosporin A (CsA) $(2 \mu \mathrm{M})$ and FK-506 $\left(\mathrm{C}_{44} \mathrm{H}_{69} \mathrm{NO}_{12}\right)$ $(2 \mu \mathrm{M})$ (Tocris Bioscience) for 30 minutes, rinsed in $\mathrm{PBS}+1 \mathrm{mM} \mathrm{Ca}^{2+}$, and lysed in ice-cold radioimmunoprecipitation assay buffer plus inhibitors [20 mM Tris, $\mathrm{pH}$ 7.4; $150 \mathrm{~mm} \mathrm{NaCl} ; 1 \%$ Triton X-100; $1 \%$ NP40; $5 \mathrm{mM}$ EDTA; $5 \mathrm{mM}$ NaF; $5 \mathrm{mM} \beta$-glycerophosphate; $2 \mathrm{mg} / \mathrm{ml}$ leupeptin; $2 \mathrm{mg} / \mathrm{ml}$ pepstatin; and $1 \mathrm{mM}$ 4-(2-aminoethyl)benzenesulfonyl fluoride]. After pelleting cell debris, $5 \%$ of each sample was set aside for input, and the remaining lysate was tumbled overnight at $4^{\circ} \mathrm{C}$ with anti-GFP antibody $(4 \mu \mathrm{g}$; A-11122; Life Technologies, Carlsbad, CA). The following day, lysates were tumbled with Pierce Protein A Sepharose beads (Thermo Fisher Scientific) for 2 hours at $4^{\circ} \mathrm{C}$. After three washes with lysis buffer, protein was eluted from beads in $2 \times$ sample buffer + DTT $(50 \mu \mathrm{M})$. Samples were then resolved using SDS-PAGE and Western blotting for phospho-MKK7 
(1:1000 in 5\% bovine serum albumin Tris-buffered saline/Tween 20; catalog number 4171; Cell Signaling Technology, Danvers, MA), antiGFP (1:1000), and anti-myc (1:100).

MKK7 Peptide-CaNA Binding Assays. N-biotinylated, C-amidated MKK7 $\gamma$ (35SPQRPRPIIVITLSPAPA52), MKK $\beta$ (35SPQRPRPTLQLPLANDGG52), PVIVIT (1GPHPVIVITGPHEE14), or control peptide (Ht31; 1LIEEAASRIVDAVIEQVK18) were custom synthesized (Biomatik, Cambridge, ON, Canada). For precipitation of CaNA-myc from HEK-293 cell extracts, $10 \mu \mathrm{g}$ of peptide was incubated with streptavidin-agarose (Sigma-Aldrich, St. Louis, MO) (20 $\mu \mathrm{l}$ of packed beads) in hypotonic buffer (10 mM Hepes, $\mathrm{pH} 7.4$; $10 \mathrm{mM} \mathrm{KCl} ; 1.5 \mathrm{mM} \mathrm{MgCl}_{2} ; 0.1 \% \mathrm{NP} 40 ; 0.1 \%$ bovine serum albumin; $1 \mathrm{mM}$ DTT; $1 \mathrm{mM}$ benzamidine; $1 \mathrm{mM}$ leupeptin; and $1 \mathrm{mM}$ AEBSF) for 4 hours at $4^{\circ} \mathrm{C}$. The slurry was centrifuged $(3000 \mathrm{~g})$, and the unbound peptide was removed. The beads were then incubated in $\sim 1 \mathrm{mg}$ of HEK-293 cell extracts expressing CaNA-myc in a total volume of $100 \mu \mathrm{l}$ overnight at $4^{\circ} \mathrm{C}$. Protein complexes were pelleted by centrifugation $(3000 \mathrm{~g})$, washed $5 \times$ with hypotonic buffer containing $1 \mathrm{M} \mathrm{NaCl}$ and $5 \times$ with hypotonic buffer, eluted with SDS-PAGE sample buffer and immunoblotted using anti-myc, as described above. The ability of MKK7 and PVIVIT peptides to compete with fluorescent PVIVIT peptide for binding to purified $\mathrm{CaN}$ was measured using fluorescence polarization as described previously (Li et al., 2004). Briefly, a complex of $1 \mu \mathrm{M}$ CaNA,B holoenzyme and $100 \mathrm{nM}$ Oregon greenlabeled PVIVIT was formed in PBS with $1 \mathrm{mg} / \mathrm{ml}$ bovine $\gamma$-globulin, followed by titration of MKK7 $\gamma$ peptide to $200 \mu \mathrm{M}$ or MKK7 $\beta$ peptide to $2000 \mu \mathrm{M}$. Competition of fluorescence polarization representing Oregongreen-PVIVIT bound to CaN was measured using a Synergy2 Plate Reader (BioTek, Winooski, VT).

Imaging of MKK7 and CaN Interaction and Localization in COS7 Cells. Living COS7 cells on 25 -mm glass coverslips were imaged at room temperature 24-48 hours post-transfection using a Nikon (Tokyo, Japan) TE-300 Inverted Microscope equipped with a $63 \times$ or $100 \times$ plan-apo/1.4 numerical aperture objective, CHROMA (Bellows Falls, VT) JP4 CFP/YFP/FRET filter sets, SensiCam digital CCD camera (The Cooke Corporation, Auburn Hills, MI), and Slidebook 4.0 software (Intelligent Imaging Innovations, Denver, CO). For three-filter (3F) micro-FRET measurements of sensitized FRET emission, CFP, YFP, and raw FRET images were captured with $2 \times 2$ binning. For measurements of FRET as CFP-donor dequenching after YFP-acceptor photobleaching (PB), YFP was bleached in a subset of the same cells with $535 \pm 20 \mathrm{~nm}$ light for 2 minutes and CFP imaged again essentially as described previously (Oliveria et al., 2003, 2007; Gorski et al., 2005). Briefly, for the 3F FRET method, YFP, CFP, and raw FRET fluorescence was detected in single $x-y$ planes in living cells using 250-millisecond exposure times to capture three images: 1) YFPexcitation/YFPemission; 2) CFPexcitation/CFPemission; and 3) CFPexcitatio$\mathrm{n} /$ YFPemission (raw FRET). After background subtraction, fractional image subtraction corrected for CFP bleed-through (0.5) and YFP crossexcitation (0.02) to yield an image of corrected, sensitized FRET (FRETc) $[$ FRETc $=$ raw FRET $-(0.5 \times \mathrm{CFP})-(0.02 \times \mathrm{YFP})]$, which was then gated to the CFP donor channel to create a FRETc/CFP pseudocolor image of relative FRET intensity in the cell. Mean CFP, YFP, and raw FRET fluorescence intensities were then measured by mask analysis of cytoplasmic regions in Slidebook 4.0 (Intelligent Imaging Innovations), as described previously (Sorkin et al., 2000; Oliveria et al., 2003, 2007). Apparent FRET efficiency (Eeff) values for sensitized FRET were calculated from these mean intensities using the following equations as described in the studies by Erickson et al. (2003) and Oliveria et al. $(2007)$ : and Eeff $=(F R-1) \times\left(\varepsilon_{\mathrm{YFP} 440} / \varepsilon_{\mathrm{CFP} 440}\right)$, where $\mathrm{FR}=($ rawFRET $-(0.5 \times \mathrm{CFP})) /(0.02 \times \mathrm{YFP})$, and $\varepsilon_{\mathrm{YFP} 440}$ and $\varepsilon_{\mathrm{CFP} 440}$ represent the average molar extinction coefficients for YFP and CFP over the band pass of the CFP excitation filter $\left(\varepsilon_{\mathrm{YFP} 440} / \varepsilon_{\mathrm{CFP} 440}=0.094\right)$. For YFP PB measurements of CFP dequenching, Eeff was independently measured and calculated as Eeff $=1-$ (CFPpre/CFPpost), where CFPpre is CFP donor intensity before and CFPpost is CFP donor intensity after. Eeff calculated from either sensitized FRET measurements or PB measurements of CFP donor deqeunching takes into account cell-to-cell variation in the expression of YFP and CFP, such that these FRET indices are effectively independent of donor and acceptor concentrations. Similar mask analysis to measure the mean CFP and YFP fluorescence intensity in regions of cytoplasm versus nucleus of individual living cells was performed to calculate the cytoplasm/nucleus mean intensity ratios presented in Figs. 5 and 6 . In Fig. 7, COS7 cells were fixed in 3.7\% formaldehyde in PBS for 5 minutes prior to imaging CFP and YFP fluorescence and measurement of cytoplasm/nucleus mean intensity ratios as above for living cells.

Quantitative real-time PCR Analysis of MKK7 $\gamma$ mRNA Expression. Total RNA ( $5 \mu \mathrm{g})$ was reverse transcribed in a volume
MKK7 35 SPQRPRPT----------------LQLPLANDGG 52 35 SPQRPRPTLQLPLANDGG 52

CaNA binding motif Stress- oxidative, UV C

Extracelluar Ligands- growth factors, hormones, transmitters

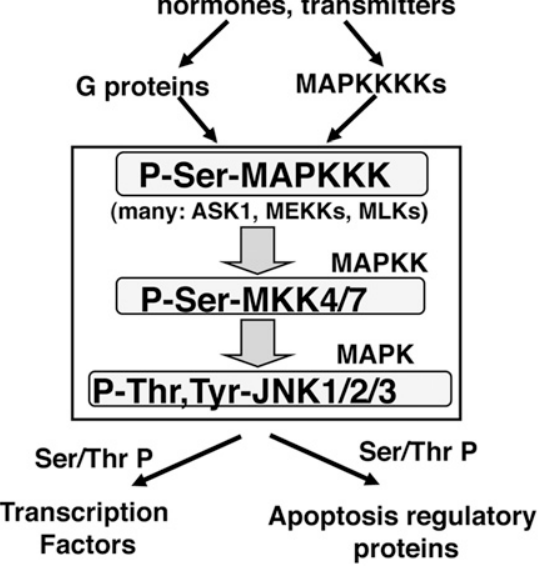

91

\begin{tabular}{|c|c|}
\hline JNK BD & Kinase Domain \\
\hline \multicolumn{2}{|c|}{$\begin{array}{c}\text { CaNA binding } \\
\text { motif }\end{array}$} \\
\hline JNK BD & Kinase Domain \\
\hline
\end{tabular}

$\operatorname{MKK} \mathbf{\alpha}$
Fig. 1. MKK7 $\gamma$ contains a putative consensus PxIxIT docking motif for CaN. (A) Sequence alignments within the $\mathrm{N}$-terminal domains of MKK7 $\gamma$ and MKK7 $\beta$ showing how alternative splicing of exon 2 introduces 17 amino acids that are unique to MKK7 $\gamma$ and contain a putative consensus PxIxIT motif, 41PIIVIT46. An alternate alignment of the MKK7 $\beta$ N-terminal domain sequence reveals a possible nonconsensus PxIxIT motif variant of PTLQLP. (B) Diagram of the role of MKK7 as an MAPK kinase (along with MKK4) in the JNKMAPK signaling pathway. MLK=Mixedlineage kinases, ASK1=Apotosis-sensing kinase 1, MEKK=Map/ERK kinase, kinases also known as MAP3Ks and MAPKKKs. (C) Diagrams of the three N-terminal $(\alpha, \beta, \gamma)$ and two C-terminal $(1,2)$ alternatively spliced isoforms of MKK7. Locations of the common kinase domain, the JNK-binding $\mathrm{N}$-terminal domain, and the potential $\mathrm{CaN}$ binding PxIxIT motifs are indicated. 


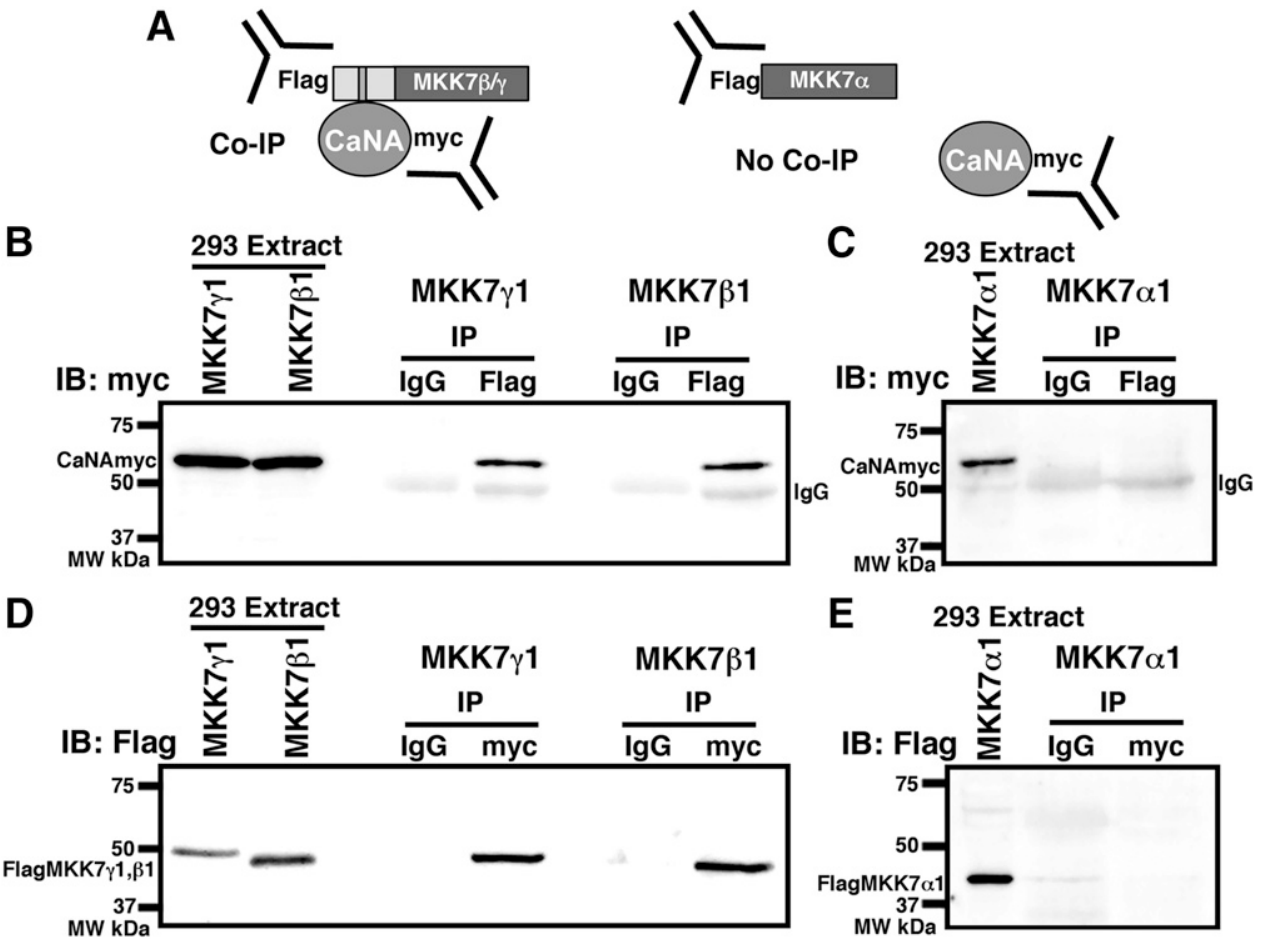

Fig. 2. MKK7 $\gamma$ and MKK7 $\beta$ coimmunoprecipitate with CaN from HEK-293 cells. (A) Diagram showing the experimental design detecting reciprocal co-IP of CaNAmyc and Flag-MKK7 $\gamma$ and Flag-MKK7 $\beta$, but not Flag-MKK7 $\alpha$ from transfected HEK-293 cells. (B) Co-IP of CaNA-myc, detected by anti-myc immunoblotting (IB) with anti-Flag pull-down of Flag-MKK7 $\gamma 1$ and Flag-MKK7 $\beta 1$ but not nonimmune rabbit IgG. (C) No co-IP of CaNA-myc with Flag-MKK $7 \alpha 1$. (D) Co-IP of Flag-MKK $7 \gamma 1$ and Flag-MKK7 $\beta 1$ detected by anti-Flag IB with anti-myc pull-down of CaNA-myc but not nonimmune mouse IgG. (E) No co-IP Flag-MKK $7 \alpha 1$ with CaNA-myc. All data are representative of three independent experiments. MW, molecular weight.

of $20 \mu \mathrm{l}$ using random hexamers and MMLV (Moloney Murine Leukemia Virus) reverse transcriptase. Aliquots $(1 \mu \mathrm{l})$ of reverse transcription reactions were subjected to PCR in $25-\mu \mathrm{l}$ reactions with SYBR Green Jumpstart Taq Readymix (Sigma-Aldrich) and the primers for $\mathrm{MKK} 7 \gamma$, forward primer 5'-ATCACTCTAAGCCCTGCTCC-3' and reverse primer 5'-TCTCTGAGGATGGTGAGCGG-3', using an I Cycler (Bio-Rad). Glyceraldehyde-3-phosphate dehydrogenase (GAPDH) mRNA levels were measured by quantitative real-time PCR (qRT-PCR) with forward primer 5'-CGTGGAGTCTACTGGCGTCTTCAC-3' and reverse primer 5'-CGGAGATGATGACCCTTTTGGC-3' in replicate samples as for the normalization of MKK $7 \gamma$ mRNA expression, and the data are presented as "relative expression."

Phospho-JNK Immunoblots and JNK Activity Assays in Rat Vascular Smooth Muscle Cells. Rat vascular smooth muscle cells (rVSMCs) cultured in Eagle's minimal essential medium containing $10 \%$ fetal bovine serum were switched to Eagle's minimal essential medium containing $0.2 \%$ fetal bovine serum and treated as indicated. After incubation, the cell monolayers were rinsed with PBS and collected in lysis buffer $(0.5 \%$ Triton X-100; $50 \mathrm{mM} \beta$-glycerophosphate, $\mathrm{pH} 7.2 ; 0.1 \mathrm{mM} \mathrm{Na} \mathrm{VO}_{4} ; 2 \mathrm{mM} \mathrm{MgCl} 2 ; 1$ mM EGTA; 1 mM DTT; $2 \mathrm{mg} / \mathrm{ml}$ leupeptin; and $4 \mathrm{mg} / \mathrm{ml}$ aprotinin). Microfuged extracts containing $200 \mu \mathrm{g}$ of protein were submitted to immunoblot analyses for phospho-JNK1/2 (1:1000; Cell Signaling Technology) or extracts were incubated $\left(4^{\circ} \mathrm{C}, 2\right.$ hours) with $2 \mu \mathrm{g}$ of anti-JNK1 antibody (C17; Santa Cruz Biotechnology) and $10 \mathrm{ml}$ of packed Protein A Sepharose in a total volume of $0.5 \mathrm{ml}$. The immune complexes were washed three times in lysis buffer and then suspended in $40 \mu \mathrm{l}$ of $50 \mathrm{mM}$ $\beta$-glycerophosphate $\left(\mathrm{pH}\right.$ 7.2), $0.1 \mathrm{mM} \mathrm{Na} \mathrm{NO}_{4}, 10 \mathrm{mM} \mathrm{MgCl}_{2}$, $100 \mu \mathrm{M}\left[\mathrm{g}_{-}{ }^{32} \mathrm{P}\right]-A T P(5000 \mathrm{cpm} / \mathrm{pmol}), 1 \mathrm{mM}$ EGTA, and $2 \mathrm{mg}$ of glutathione $S$-transferase (GST)-cJun(1-79). After a 20 -minute incubation at $30^{\circ} \mathrm{C}$, the kinase reactions were terminated with $10 \mu \mathrm{l}$ of SDS sample buffer and submitted to SDS-PAGE. The GST-cJun(1-79) polypeptides were excised from the Coomassie-stained and dried gels, and the incorporated radioactivity was determined in a scintillation counter.

Statistical Analyses. All graphs in the figures represent the mean \pm S.E., and $P$ values were determined as indicated in the figure legends by unpaired two-tailed Student's $t$ test in Excel for Mac
2011 version 14.7 .7 (Microsoft, Redmond, WA), by one-sample $t$ test in Prism versions 4.0-6.0 (GraphPad Software, La Jolla, CA), or by oneway ANOVA with Bonferroni post hoc correction for multiple comparisons in Prism versions 4.0-6.0. A value of $P<0.05$ is considered significant unless otherwise noted in the figure legends because of additional adjustment for multiple comparisons.

\section{Results}

To identify novel signaling proteins that may bind $\mathrm{CaN}$, we used the program BLASTP (http://blast.ncbi.nlm.nih.gov) to search all nonredundant mouse, rat, and human protein sequences in GenBank, Protein Data Bank, SWISS-PROT, Protein Information Resource, and Protein Research Foundation for potential high-affinity PxIxIT motifs similar in sequence to PVIVIT or IAIIIT from AKAP79. The top hit in the search for sequences similar to PVIVIT identified residues 41PIIVIT46 in N-terminal domain of the $\gamma$ isoform of MKK7 that conforms to a consensus PxIxIT motif and differs from PVIVIT by only a single substitution of $\mathrm{I}$ for $\mathrm{V}$ in the nonconserved second position (Fig. 1A). Importantly, this 41PIIVIT46 sequence is identical in the MKK7 $\gamma$ sequences for humans, mice, and rats. The JNK-MAPK pathway is activated by a number of different cellular stresses and receptors binding extracellular ligands (Fig. 1B). In these pathways, MKK7 kinase activity is activated by Ser and Thr phosphorylation in its activation loop by a number of different upstream MAPK kinase kinases (MAPKKKs) and then activates $\mathrm{JNK} 1 / 2 / 3$ kinase activity through phosphorylation on Thr and Tyr residues in the JNK activation loop to then promote downstream phosphorylation of targets that control a variety of processes including transcription and apoptosis (Fig. 1B) (Davies and Tournier, 2012). MKK7 $\gamma$ is one of three isoforms of MKK7 ( $\alpha, \beta$, and $\gamma$ ) that differ in their N-terminal regions because of alternative splicing (Tournier et al., 1999) 


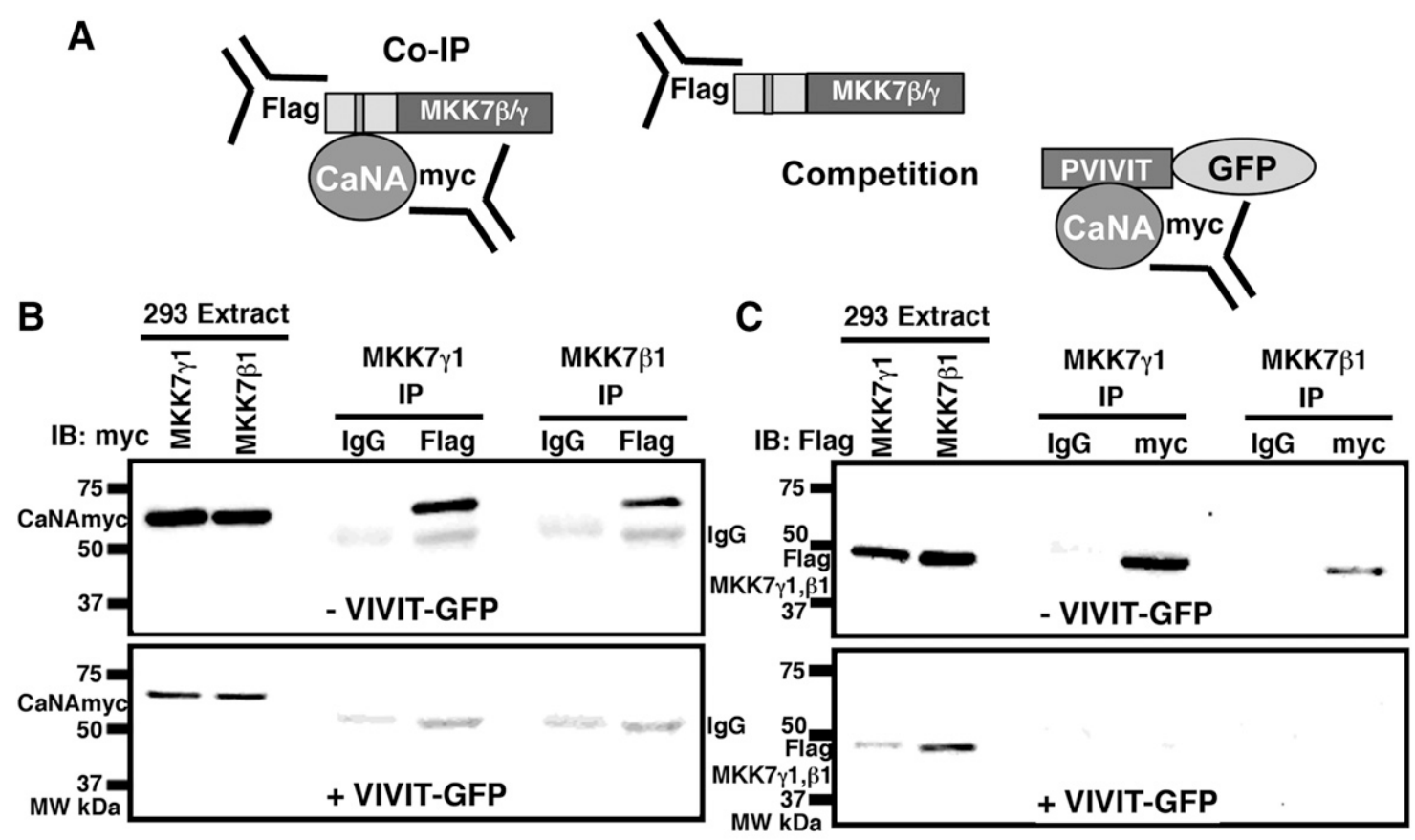

D 293 Extracts

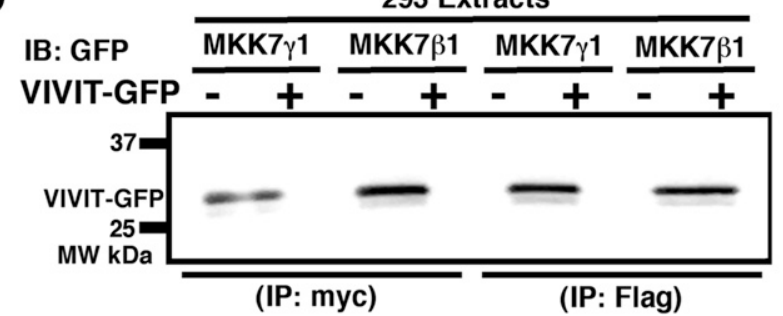

Fig. 3. A competing PxIxIT motif PVIVIT prevents CaN co-IP with MKK7 $\gamma$ and MKK7 $\beta$. (A) Diagram showing the experimental design for detecting PVIVIT-GFP disruption of reciprocal co-IP between CaNA-myc and Flag-MKK7 $\gamma$ and Flag-MKK7 $\beta$ in HEK-293 cells. (B) Expression of PVIVIT (bottom panel, +VIVIT-GFP) prevents normal anti-Flag co-IP of CaNA-myc with Flag-MKK7 $\gamma 1$ and Flag-MKK7 $\beta 1$ (top panel, -VIVIT-GFP). (C) Expression of PVIVIT (bottom panel, +VIVIT-GFP) prevents normal anti-myc co-IP of Flag-MKK7 $\gamma 1$ and Flag-MKK7 $\beta 1$ with CaNA-myc (top panel, -VIVIT-GFP). (D) Anti-GFP immunoblotting (IB) detecting PVIVIT-GFP expression in the extracts used for the anti-Flag and anti-myc IP in (B) and (C). All data are representative of three independent experiments. MW, molecular weight.

(Fig. 1C). The $\beta$ and $\gamma$ isoforms both possess an $\mathrm{N}$-terminal regulatory domain (residues 1-91 in MKK7 $\gamma$ ) appended to the kinase domain that contains docking sites for its downstream substrate/target JNK (Tournier et al., 1999). In contrast, MKK7 $\alpha$ lacks this N-terminal regulatory domain (Fig. 1C). Closer inspection of the MKK7 gene structure reveals that the 41PIIVIT46 sequence is contained within a 17 -amino acid region encoded by exon 2 that is unique to $\operatorname{MKK} 7 \gamma$; thus, MKK7 $\beta$ contains an alternatively spliced sequence in this region of 41PTLQLP46, which has, at best, weak homology to a PxIxIT motif, with only one consensus residue at P41, conservative substitutions of I for $\mathrm{L}$ at positions 43 and 45 , and a nonconservative substitution of $\mathrm{T}$ for $\mathrm{P}$ at position 46 (Fig. 1A). However, as mentioned above, since there is considerable variation around the PxIxIT consensus motif in known CaN binding partners, both MKK7 $\gamma$ and $\beta$ could potentially interact with $\mathrm{CaN}$, albeit likely with different affinities.

To test for interactions between $\mathrm{CaN}$ and the different MKK7 isoforms, we transfected HEK-293 cells with C-terminal myc-tagged CaNA and N-terminal Flag-tagged MKK $7 \alpha, \beta$, or $\gamma$, and performed reciprocal co-IP experiments
(Fig. 2A). There is also alternative splicing of the MKK7 $\mathrm{C}$-terminal region generating a shorter and longer C-terminal version for each $\mathrm{N}$-terminal variant (i.e., MKK $7 \gamma 1$ vs. MKK7 $\gamma 2$ ) (Fig. 1C), giving rise to a total of six different MKK7 isoforms (Tournier et al., 1999); however, we confined our analysis to the shorter $\alpha 1, \beta 1$, and $\gamma 1$ isoforms. Anti-Flag IP of MKK $\gamma$ led to coprecipitation of CaNA-myc as predicted based on the presence of the strong, consensus PxIxIT motif (Fig. 2B). Somewhat surprisingly, anti-Flag IP of MKK7 $\beta$ also pulled down CaNA-myc (Fig. 2B), suggesting that both MKK $\gamma$ and MKK7 $\beta$ may be present in complexes with $\mathrm{CaN}$ in cells. However, anti-Flag IP of the shorter MKK $7 \alpha$ isoform lacking the entire $\mathrm{N}$-terminal domain failed to precipitate $\mathrm{CaN}$ (Fig. 2, A and C). Likewise, anti-myc IP of CaNA led to co-IP of both MKK7 $\beta$ and MKK $\gamma$ (Fig. 2D), but not to MKK7 $\alpha$ (Fig. 2E). Nonimmune IgG control IP also failed to precipitate FlagMKK7 or CaNA-myc under any of the above conditions, further demonstrating specificity of the interactions of CaNA with MKK7 $\beta$ and MKK7 $\gamma$.

To test whether the formation of complexes containing CaN and MKK7 $\beta$ and $\gamma$ in HEK-293 cells involve CaNA docking to PxIxIT motifs, we overexpressed PVIVIT-GFP as a 
A

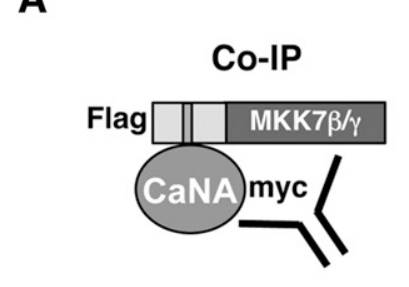

Flag \begin{tabular}{l||l|l|}
\hline & $M K K 7 \beta / \gamma$ \\
\hline
\end{tabular}

Competition

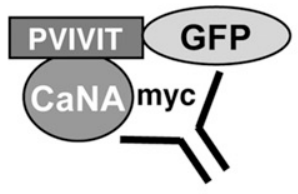

B
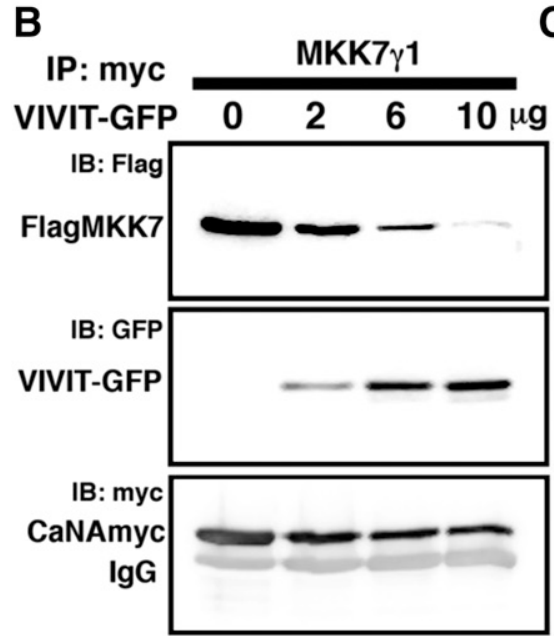

C
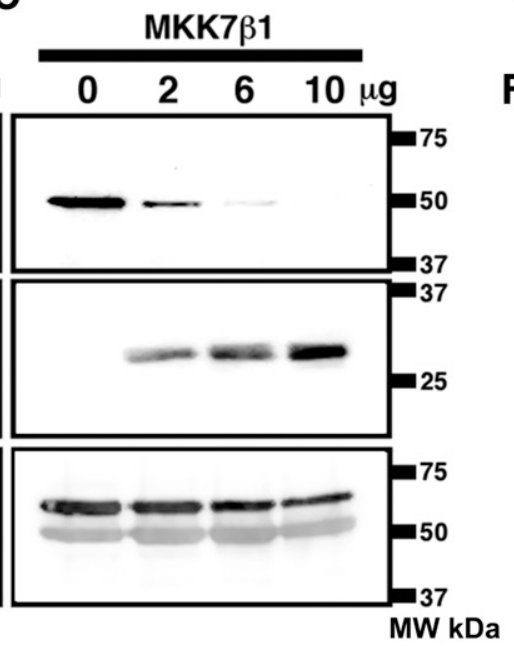

D
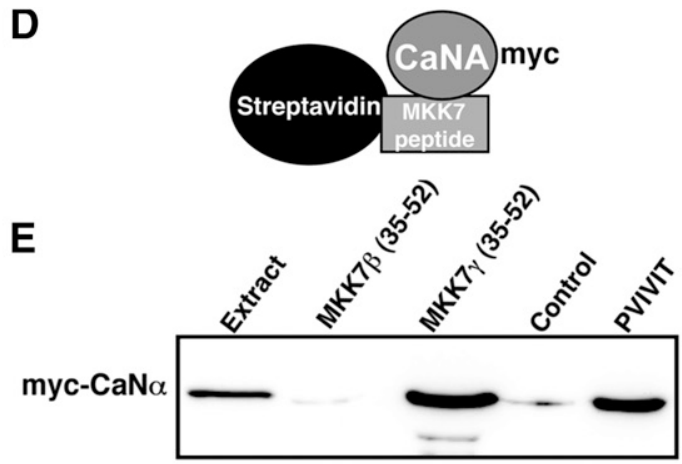

$\mathbf{F}$

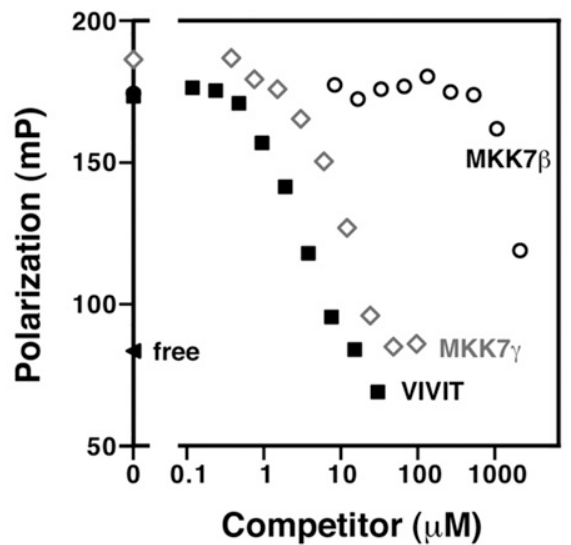

Fig. 4. MKK7 $\gamma$, but not MKK7 $\beta$, binds to CaN through a direct PxIxIT motif interaction. (A) Diagram showing the experimental design for detecting PVIVIT-GFP disruption of anti-myc co-IP of CaNA-myc and Flag-MKK7 $\gamma$ and -MKK7 $\beta$ in HEK-293 cells. Transfection of HEK-293 cells with higher amounts of plasmid DNA leading to higher levels of VIVIT-GFP expression [middle panel, immunoblotting (IB): GFP] are required to disrupt anti-myc co-IP of Flag-MKK7 $\gamma 1$ (B) versus Flag-MKK7 $\beta 1$ (C) (top panel, IB: Flag) with CaNA-myc (bottom panel; IB: myc). (D) Diagram showing the experimental design for detecting precipitation of CaNA-myc by biotinylated MKK7 $\beta$ or MKK7 $\gamma$ 35-52 peptides coupled to streptavidin agarose beads. (E) MKK7 $\gamma$ 35-52 and PVIVIT biotinylated peptides precipitate CaNA-myc from HEK-293 cell extracts. MKK7 $\beta$ 35-52 and negative control (Ht31) biotinylated peptides do not precipitate CaNA-myc. (F) The MKK7 $\gamma$ 35-52 peptide competes with an affinity similar to PVIVIT for fluorescent-PVIVIT binding to CaN measured by fluorescence polarization $(\mathrm{mP})$. MKK7 $\beta 35-52$ only competes for fluorescent PVIVIT binding to CaN at much higher concentrations. All data are representative of three independent experiments.

high-affinity competing PxIxIT binding partner for CaNA (Fig. 3A). Accordingly, anti-FLAG co-IP of CaNA-myc with MKK $7 \beta$ or MKK $\gamma$ (Fig. 3B) and anti-myc co-IP of Flag-MKK $\beta$ or $\mathrm{MKK} \gamma$ with CaNA-myc (Fig. 3C) were all prevented by VIVIT-GFP overexpression (Fig. 3D). Thus, coprecipitation of CaNA with $\mathrm{MKK} \gamma$, as well as MKK7 $\beta$, requires the PxIxIT motif docking site on CaNA where PVIVIT binds. Next, we began to evaluate the relative affinities of the PxIxIT site interactions of $\mathrm{CaN}$ in complexes with $\mathrm{MKK} \gamma$ and MKK7 $\beta$ by varying the amount of expression of the competing PVIVITGFP protein (Fig. 4, A and B). Consistent with a potentially higher affinity PxIxIT interaction between CaNA and MKK $\gamma$ compared with MKK7 $\beta$, higher levels of VIVIT-GFP expression were required to prevent anti-myc CaNA co-IP of FlagMKK7 $\gamma$ than for Flag-MKK7 $\beta$ (Fig. 4B).

However, co-IP of CaN with MKK7 $\beta$ and/or MKK7 $\gamma$ from cell extracts could be mediated either by direct binding to the predicted PxIxIT motifs in these proteins or by indirect interaction through unknown PxIxIT-containing proteins that somehow associate with the $\mathrm{MKK} 7 \beta / \gamma$ N-terminal domain. Thus, to assay for direct binding between $\mathrm{CaN}$ and the predicted PxIxIT motifs in MKK7 $\gamma$ and $\beta$, we synthesized $\mathrm{N}$-biotinylated 18-amino acid peptides corresponding to residues 35-52 containing the MKK7 $\gamma$ PIIVIT and MKK7 $\beta$ PTLQLP sequences, coupled them to streptavidin-agarose beads, and then tested these peptides for coprecipitation of CaNA-myc from HEK-293 cell extracts (Fig. 4, D and E). As a positive control in this assay, we used a biotinylated 14-amino acid peptide encoding the PVIVIT sequence (Aramburu et al., 1999), and as negative control we used a biotinylated 18amino acid Ht31 peptide, that binds to PKA but not CaN (Carr et al., 1992; Dell'Acqua et al., 2002). Although the MKK7 $\gamma$ peptide pulled down CaNA-myc to a similar extent as the PVIVIT positive control peptide, the MKK7 $\beta$ peptide behaved in a manner similar to that of the Ht31 negative control peptide and failed to precipitate CaNA-myc (Fig. 4E). Last, we assayed for direct binding of the MKK7 $\gamma$ versus $\beta$ 35-52 peptides to purified CaN in vitro by competition with PVIVIT. In this assay, CaN binding to fluorescently labeled VIVIT peptide was detected by increased fluorescence polarization (anisotropy) due to formation of the larger, slower rotating CaN-peptide complex compared with the free peptide (Fig. 4F) (Li et al., 2004). Increasing concentrations of unlabeled MKK7 $\gamma$, MKK7 $\beta$, or VIVIT peptides were added to compete with fluorescent VIVIT binding, causing a decrease in the polarization signal (Fig. 4F). In this assay, the MKK7 $\gamma$ peptide competed with an $\mathrm{IC}_{50}$ of $\sim 10 \mu \mathrm{M}$, which was nearly as effective as unlabeled VIVIT peptide competing against itself. In contrast, the MKK7 $\beta$ peptide showed at best partial competition of fluorescent VIVIT binding and only at much 


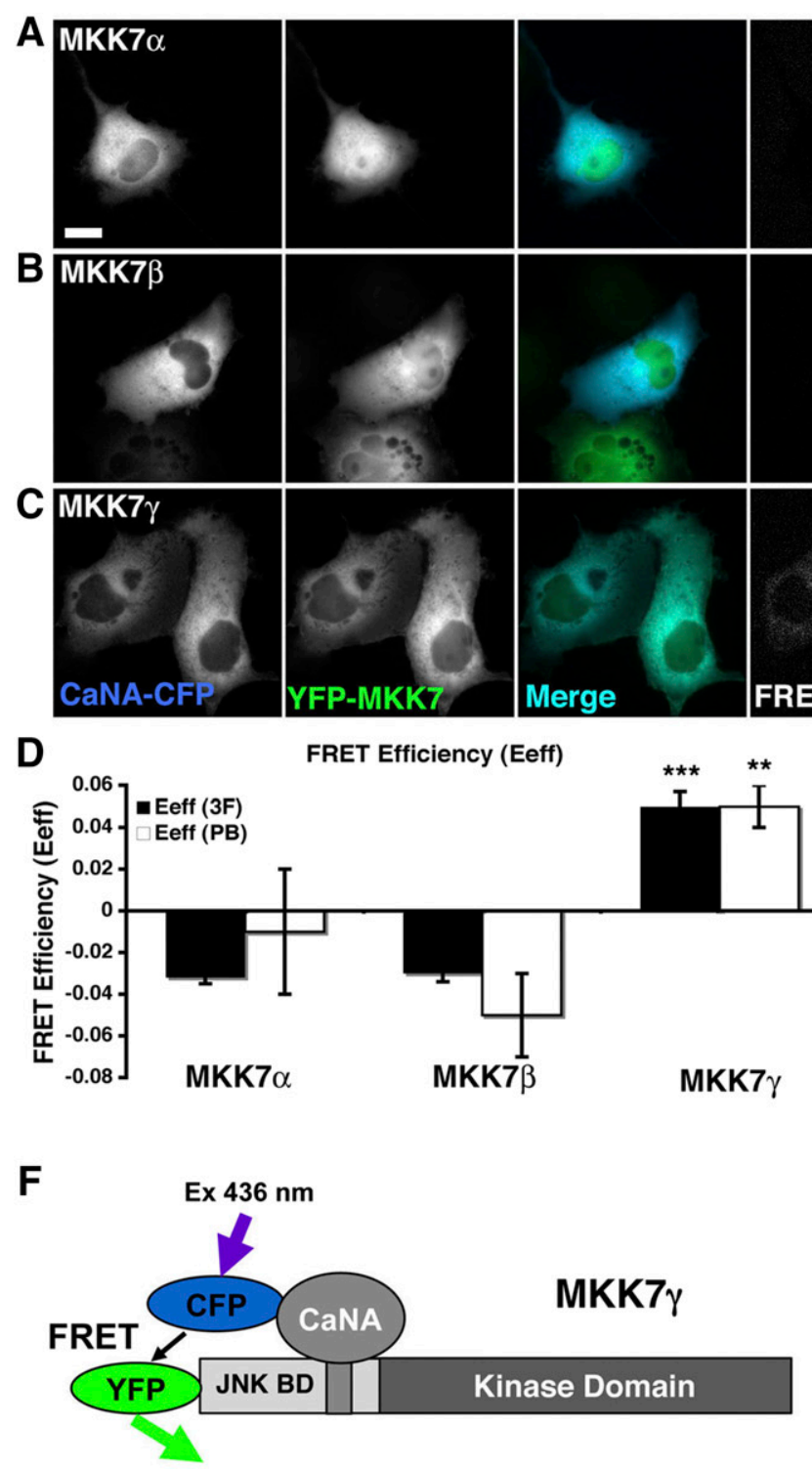

Em $535 \mathrm{~nm}$
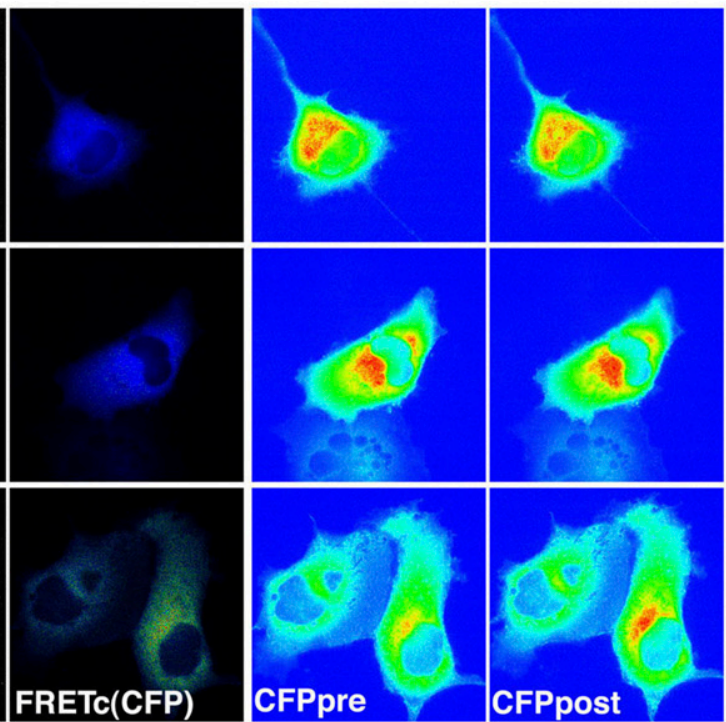

E

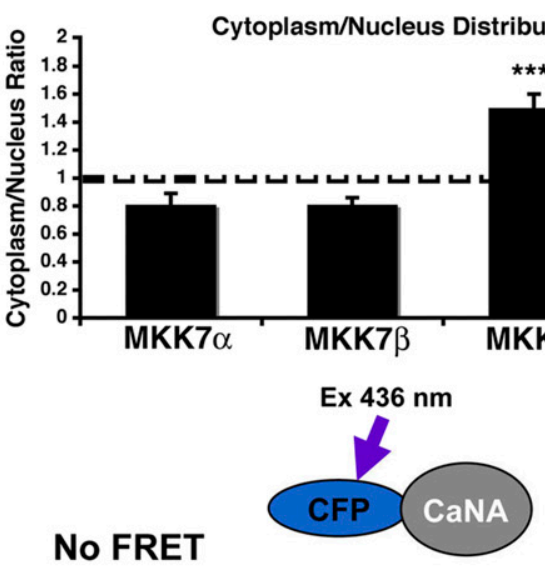

MKK7 $\beta$

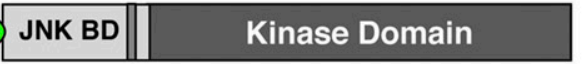

$\operatorname{MKK} 7 \alpha$

YFP

Fig. 5. Interaction between MKK7 $\gamma$ and CaN imaged by FRET microscopy in the cytoplasm of living cells. Sensitized YFP acceptor 3F FRET emission (FRETc) or CFP donor dequenching after PB of the YFP acceptor (CFPpost $>$ CFPpre) is not detected for CaNA-CFP with YFP-MKK7 $\alpha$ (A) or YFPMKK7 $\beta$ (B) but is detected with YFP-MKK7 $\gamma(\mathrm{C})$ in living COS7 cells. The merge panels show overlap (turquoise) of CaNA-CFP (blue) and YFP-MKK7 (green) localization in the cytoplasm but not in the nucleus. FRETc is shown in both monochrome and in pseudocolor on the same relative intensity scales (blue = no FRETc to green, yellow, orange; and red = high with FRETc gated to the CFP donor channel). For YFP PB FRET, CFPpre and CFPpost are shown on the same relative pseudocolor intensity scale (blue = no intensity to red = high intensity). Scale bar, $10 \mu \mathrm{m}$. (D) Quantification of sensitized $3 \mathrm{~F}$ FRET emission and YFP PB CFP dequenching as apparent Eeff for multiple cells showing significant FRET for MKK7 $\gamma$ [Eeff $(3 F) 0.050 \pm 0.007, n=12$; *** $P<0.001$ by one-way ANOVA with Bonferroni post hoc correction for multiple comparisons; Eeff (PB) $0.055 \pm 0.013, n=8$; $* * P<0.01$ by one-way ANOVA with Bonferroni post hoc correction for multiple comparisons] compared with MKK7 $\alpha$ (Eeff ( 3 F) $-0.032 \pm 0.003, n=14$; Eeff (PB) $-0.022 \pm$ $0.016, n=10)$ and MKK7 $\beta$ [Eeff (3F) $-0.030 \pm 0.004, n=14$; Eeff (PB) $-0.034 \pm 0.021, n=12$ ]. Eeff values $\leq 0$ reflect a lack of FRET. (E) Quantification of cytoplasm/nucleus fluorescence intensity ratios for multiple cells showing enrichment in the cytoplasm over the nucleus $(>1.0)$ for MKK7 $\gamma(1.5 \pm 0.1$, $n=12,8 ; * * * P<0.001$ by one-way ANOVA with Bonferroni post hoc correction for multiple comparisons) and CaNA $(1.63 \pm 0.09, n=14 ; * * * P<0.001$ by one-way ANOVA with Bonferroni post hoc correction for multiple comparisons) compared with MKK7 $\alpha(0.81 \pm 0.05, n=14)$ and MKK7 $\beta(0.81 \pm 0.05, n=14)$. Diagram depicting CFP-YFP FRET between CaNA-CFP bound to the N-terminal domain of YFP-MKK7 $\gamma$ (F) but not YFP-MKK7 $\alpha$ or YFP-MKK7 $\beta$ (G). All graphs represent the mean \pm S.E. Please note that all of the $P$ values above are below a threshold of $0.05 / 3=0.0167$ that incorporates additional correction for multiple testing due to applying two different CFP-YFP FRET methods and measuring CFP and YFP cytoplasm/nucleus ratios in the same cells.

higher doses approaching $\sim 1 \mathrm{mM}$ (Fig. 4F). Based on the known $\mathrm{K}_{\mathrm{d}}$ value of $\sim 0.5 \mu \mathrm{M}$ for the VIVIT-CaN interaction, we can estimate that the MKK7 $\gamma$ PIIVIT consensus PxIxIT motif binds to $\mathrm{CaN}$ with an affinity in the physiologically relevant low-micromolar range. In contrast, the MKK7 $\beta$ PTLQLP nonconsensus sequence engages in little or no direct interaction with $\mathrm{CaN}$ except at very high concentrations that may be nonphysiologic (i.e., $>1 \mathrm{mM}$ )

These in vitro CaN binding results indicate that MKK7 $\gamma$ engages in a reasonably high-affinity direct PxIxIT site interaction with $\mathrm{CaN}$ that could easily account for the co-IP with CaNA from cell extracts observed above. However, the 


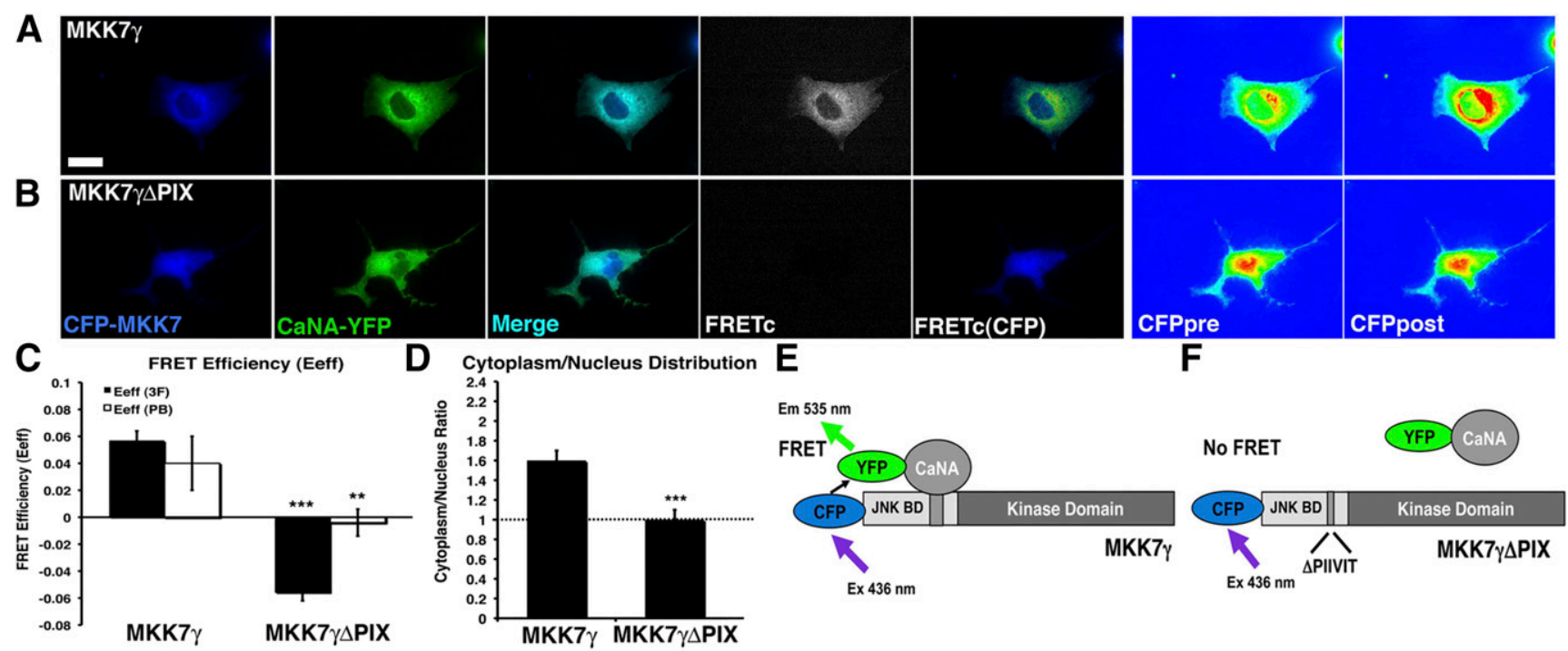

Fig. 6. Deletion of the MKK7 $\gamma$ PxIxIT motif eliminates FRET with CaN and promotes localization to the nucleus. Sensitized YFP acceptor $3 F$ FRET emission (FRETc) or CFP donor dequenching after PB of the YFP acceptor (CFPpost $>$ CFPpre) is detected for CaNA-YFP with CFP-MKK7 $\gamma$ (A) but not CFP-MKK7 $\gamma \Delta$ PIX (B) in living COS7 cells. The merge panels show overlap (turquoise) of CaNA-YFP (green) and CFP-MKK7 $\gamma$ (blue) localization in the cytoplasm but not the nucleus. FRETc is shown in both monochrome and in pseudocolor on the same relative intensity scales (blue = no FRETc to green, yellow, orange; and red = high with FRETc gated to the CFP donor channel). For YFP PB FRET, CFPpre and CFPpost values are shown on the same relative pseudocolor intensity scale (blue = no intensity to red = high intensity). Scale bar, $10 \mu \mathrm{m}$. (C) Quantification of apparent Eeff from sensitized 3F FRET emission (Eeff) and YFP PB CFP dequenching [Eeff (PB)] for multiple cells showing significant FRET for MKK7 $\gamma$ [Eeff (3F)] $0.057 \pm 0.007, n=9$; *** $P<0.001$ by unpaired, two-tailed $t$ test; Eeff (PB) $0.042 \pm 0.018, n=9 ; * * P<0.01$ by unpaired, two-tailed $t$ test] compared with MKK7 MPIX $\gamma$ [Eeff (3F) $-0.056 \pm 0.006, n=10$; Eeff (PB) $-0.004 \pm 0.01, n=10]$. Eeff values $\leq 0$ reflect lack of FRET. (D) Quantification of cytoplasm/nucleus fluorescence intensity ratios for multiple cells showing enrichment in the cytoplasm $(>1.0)$ for MKK7 $\gamma(1.55 \pm 0.07, n=9)$ but equal distribution in the nucleus and cytoplasm ( 1.0) for MKK7 $\gamma \Delta$ PIX $(1.08 \pm 0.09, n=10$; *** $P=0.0008$ by unpaired two-tailed $t$ test). Diagram depicting CFP-YFP FRET between CaNAYFP bound to the N-terminal domain of CFP-MKK7 $\gamma(\mathrm{E})$ but not to CFP-MKK7 $\gamma \Delta \mathrm{PIX}(\mathrm{F})$. All graphs represent the mean \pm S.E. Please note that all of the $P$ values above are below a threshold of $0.05 / 3=0.0167$ that incorporates additional correction for multiple testing due to applying two different CFP-YFP FRET methods and measuring CFP and YFP cytoplasm/nucleus ratios in the same cells.

best very low-affinity interaction detected for the MKK7 $\beta$ peptide with $\mathrm{CaN}$ indicates that co-IP of these proteins from cells is likely indirect and must be mediated by additional protein interactions in the complex. Thus, we next turned to FRET microscopy to measure possible direct interactions between CaNA and MKK7 $\gamma$ versus MKK7 $\beta$ in living cells. We expressed CaNA C-terminally tagged with CFP and MKK $7 \alpha$ (Fig. 5, A and G), MKK7 $\beta$ (Fig. 5, B and G), or MKK7 $\gamma$ (Fig. 5, C and F) N-terminally tagged with YFP in COS7 cells. As in our past FRET analysis of the AKAP79-CaNA interaction in living cells (Oliveria et al., 2003, 2007), we measured FRET between the CaNA-CFP and YFP-MKK7 by two independent intensity-based FRET methods: $3 \mathrm{~F}$ microFRET to measure sensitized FRET emission from the YFP acceptor (FRETc); and YFP PB to measure CFP donor dequenching (CFPpost $>$ CFPpre). For cells expressing YFPMKK7 $\alpha$ (Fig. 5A) or YFP-MKK7 $\beta$ (Fig. 5B), no FRET with CaNA-CFP was detected in corrected images of sensitized FRET [FRETc or FRETc(CFP) gated to the CFP donor channel], and no dequenching of CaNA-CFP fluorescence was seen after YFP PB (CFPpost CFPpre). In contrast, both FRETc signals and YFP PB induced increases in CFPpost $>$ CFPpre fluorescence that were observed in the cytoplasm of cells expressing YFP-MKK7 $\gamma$ and CaNA-CFP (Fig. 5C). Quantification from multiple cells of sensitized 3F FRET as apparent Eeff (Fig. 5D) (see Materials and Methods) all confirmed significant FRET between YFP-MKK7 $\gamma$ and CaNA-CFP in the cytoplasm and a lack of FRET for MKK7 $\alpha$ and MKK7 $\beta$. In addition, calculation of Eeff from YFP PB measurements of CFP dequenching independently confirmed significant FRET specifically between YFP-MKK7 $\gamma$ and CaNA-CFP (Fig. 5E). Thus, these FRET measurements in living cells are in agreement with the in vitro binding studies above and provide additional evidence in support of a direct, high-affinity interaction between $\mathrm{CaN}$ and $\mathrm{MKK} 7 \gamma$, but not MKK $7 \beta$ or MKK $7 \alpha$.

Another notable observation of these imaging experiments was that YFP-MKK7 $\alpha$ (Fig. 5A) and MKK7 $\beta$ (Fig. 5B) were localized in both the nucleus and the cytoplasm, but with greater accumulation in the nucleus. In contrast, YFP-MKK7 $\gamma$ was predominantly found in the cytoplasm with CaNA-CFP, where FRET between these two proteins was also detected (Fig. 5C). Independent quantification of cytoplasm to nucleus fluorescence intensity ratios confirmed this differential distribution of MKK7 isoforms, with MKK7 $\gamma$ and CaNA exhibiting ratios of $>1.0$, indicating cytoplasmic enrichment; and MKK $7 \alpha$ and MKK7 $\beta$ both showing ratios of $<1.0$, indicating nuclear enrichment (Fig. 5E). Thus, these imaging results indicate that MKK7 $\gamma$ binds directly to CaNA in the cytoplasm, whereas MKK7 $\alpha$ and MKK7 $\beta$, which do not directly interact with CaNA are more prevalent in the nucleus. Overall, these results reinforce the conclusion that $\mathrm{CaN}$ co-IP with MKK7 $\beta$ in Figs. 2-4 is due to $\mathrm{CaN}$ binding to additional proteins that may associate through the MKK7 N-terminal domain that is absent in MKK $7 \alpha$. In addition, given the observed differences in localization of $\mathrm{MKK} 7 \beta$ and $\mathrm{CaN}$ along with the lack of FRET, the interaction seen in lysates by co-IP may not be relevant in intact cells.

To test the specific role of the MKK7 $\gamma$ consensus PxIxIT motif in mediating localization and FRET with $\mathrm{CaN}$ in the 
A
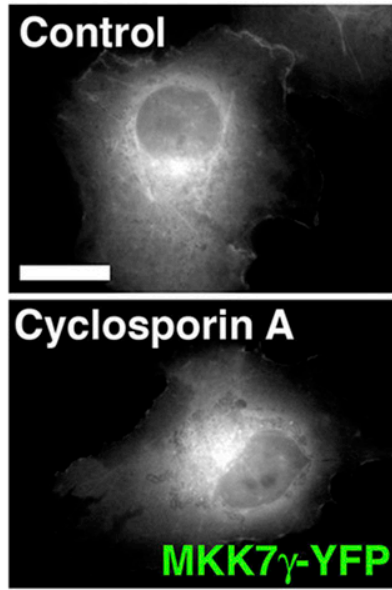

B
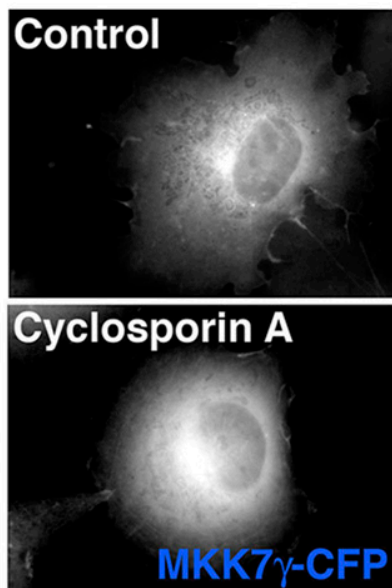

C

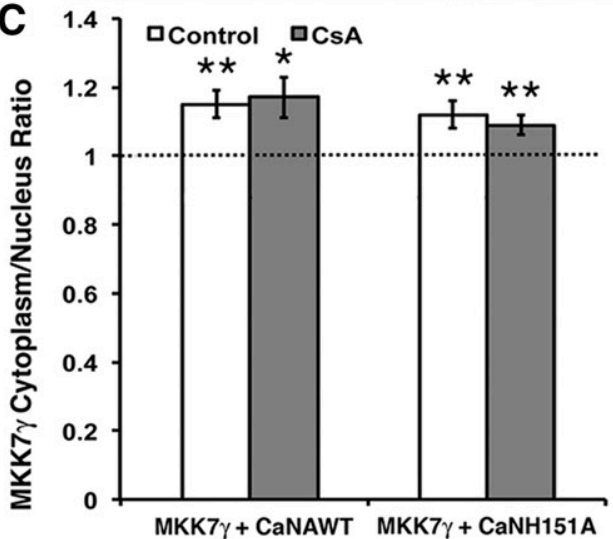

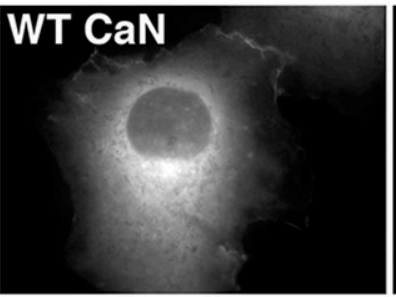
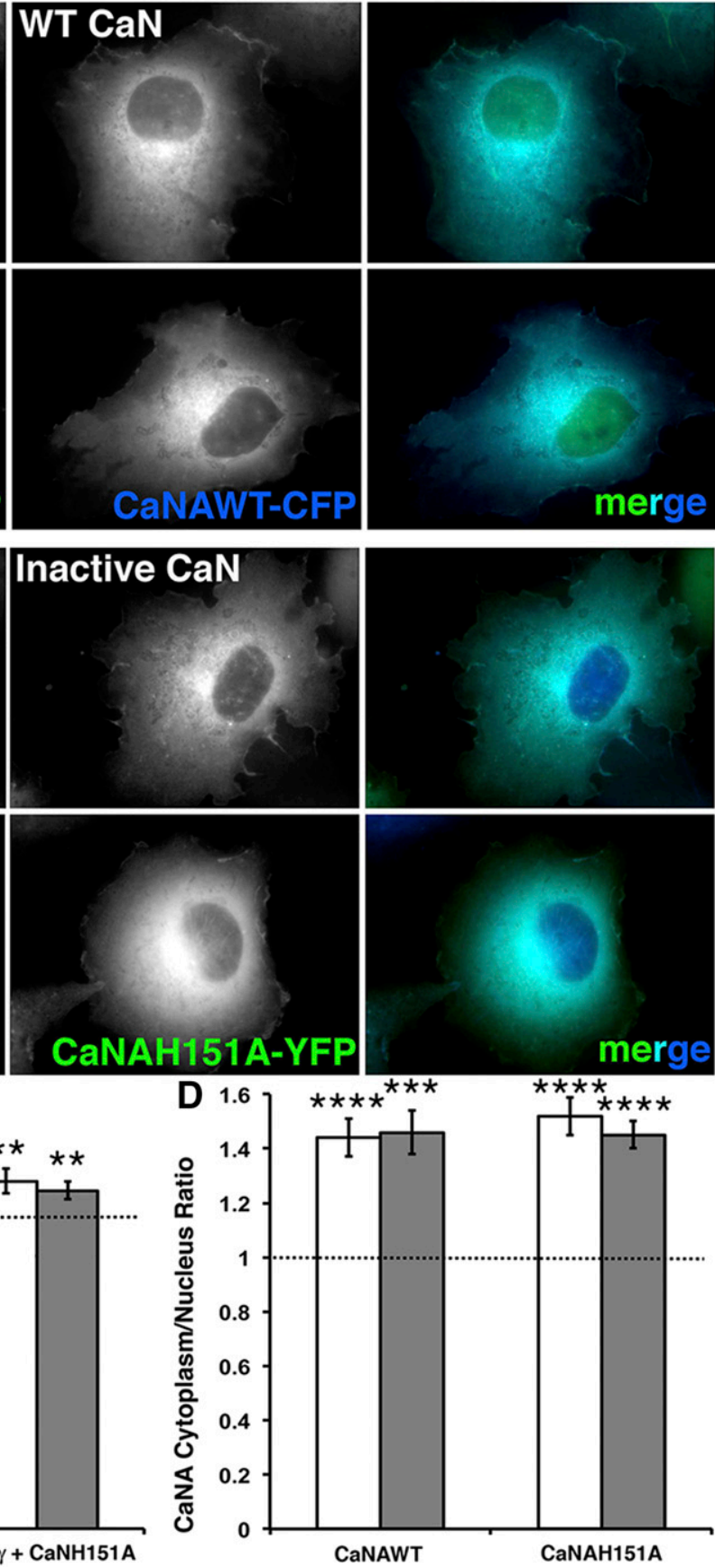

Fig. 7. CaN phosphatase activity is not required to retain $\mathrm{MKK} 7 \gamma$ in the cytoplasm. Images of fixed COS7 cells expressing YFPMKK7 $\gamma$ (green) and CaNAWT-CFP (blue) (A) or CFP-MKK7 $\gamma$ (blue) and catalytically inactive CaNH151A-YFP (green) (B) under control conditions or after treatment with the CaN phosphatase inhibitor CsA $(1 \mu \mathrm{M})$ as indicated. The merge panels show overlap (turquoise) of CaNA and MKK7 $\gamma$ localization in the cytoplasm but not the nucleus. Scale bar, $10 \mu \mathrm{m}$. Quantification of cytoplasm/ nucleus fluorescence intensity ratios for multiple cells showing maintenance of MKK7 $\gamma$ cytoplasmic enrichment [cytoplasm/ nucleus ratios all $>1.0$ by one-sample $t$ test: MKK7 $\gamma+$ CaNAWT: $1.15 \pm 0.03, n=10$, $* * P=0.0014 ; \mathrm{MKK} 7 \gamma+\mathrm{CaNAWT}+\mathrm{CsA}:$ $1.17 \pm 0.06, n=10, * P=0.020 ;$ MKK $7 \gamma$ +CaNAH151A: $1.12 \pm 0.03, n=13, * * P=$ 0.0024; MKK7 $\gamma+\mathrm{CaNAH} 151 \mathrm{~A}+\mathrm{CsA}: 1.09 \pm$ $0.03 n=11$, **P $=0.0094$; but all cytoplasm/ nucleus ratios not different for any pairwise comparisons, $P>0.05$ (n.s.) by one-way ANOVA with Bonferroni post hoc correction for multiple comparisons] (C) and CaNA cytoplasmic enrichment [cytoplasm/nucleus ratios $>1.0$ by one-sample $t$ test; CaNAWT: $1.44 \pm 0.06, n=10, * * * * P<0.0001$ CaNAWT+CsA: $1.46 \pm 0.08, n=10, * * * P=$ 0.0003; CaNAH151A: $1.52 \pm 0.07, n=13$, $* * * * P<0.0001$; CaNAH151A: $1.45 \pm 0.05$, $n=11$, **** $P<0.0001$; but all cytoplasm/ nucleus ratios are not different for any pairwise comparisons, $P>0.05$ (n.s.) by one-way ANOVA with Bonferroni post hoc correction for multiple comparisons for all conditions] (D). These cytoplasm/nucleus ratios in fixed COS7 cells for both MKK7 $\gamma$ and CaNA are somewhat lower than in live cells (Fig. 5G; Fig. 6F), likely due to some loss of cytoplasmic fluorescent protein fluorescence that occurs after fixation (Schnell et al., 2012). cytoplasm, we introduced an in-frame deletion of residues 41PIIVIT46 to create a CFP-MKK7 $\gamma \Delta$ PIX mutant (Fig. 6, A, $\mathrm{B}, \mathrm{E}$, and F). As described above for YFP-MKK $\gamma$ FRET with CaNA-CFP, CFP-MKK7 $\gamma$ and CaNA-YFP exhibited significant cytoplasmic FRETc and CFPpost $\mathrm{PB}$ dequenching signals in the cytoplasm of living COS7 cells (Fig. 6A). In contrast, the CFP-MKK7 $\gamma \Delta$ PIX mutant failed to show any FRET with CaNA-YFP and was now clearly present in both the nucleus and the cytoplasm (Fig. 6B). Quantification of Eeff from sensitized 3F FRET measurements and independently from YFP PB measurements of CFP dequenching for multiple cells both confirmed a lack of FRET between CFP-MKK $7 \gamma \Delta$ PIX and CaNA-YFP (Fig. 6C). Additional measurements of cytoplasm/nucleus ratios confirmed significantly reduced localization of MKK $7 \gamma \Delta \mathrm{PIX}$ in the cytoplasm compared with MKK7 $\gamma$ wild-type and CaN (Fig. 6D). Indeed, the cytoplasm/nucleus ratio for CFP-MKK7 $\gamma \Delta$ PIX was $\sim 1.0$, indicating equal distribution between the cytoplasm and nucleus. Thus, the PIIVIT consensus PxIxIT motif in MKK7 $\gamma$ is required for direct binding to CaNA in the cytoplasm detected as FRET in living cells (Fig. 6, E and F) and also prevents MKK7 $\gamma$ from localizing more strongly in the nucleus like the MKK7 $\alpha$ and $\beta$ isoforms, which do not interact directly with CaN.

Next, we used the CaN inhibitor CsA to examine whether the phosphatase activity of $\mathrm{CaN}$ is required for cytoplasmic retention of MKK $7 \gamma$. However, the treatment of cells coexpressing YFP-MKK7 $\gamma$ and CaNA-WT-CFP with CsA (Fig. 7A) did not alter the cytoplasmic/nucleus localization ratios for 


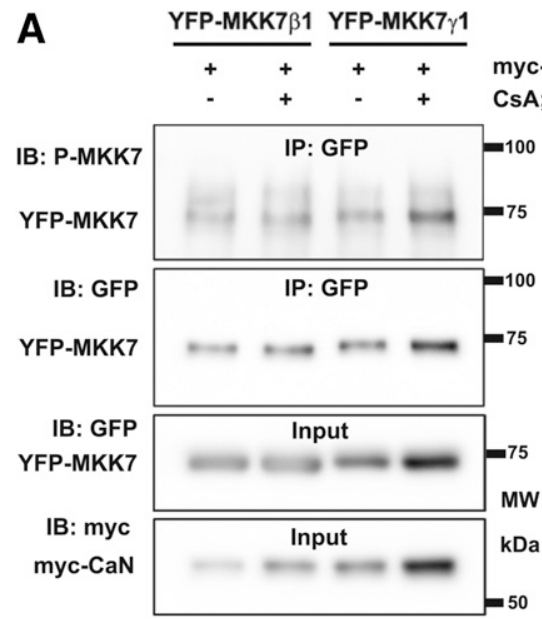

\section{B}

CsA; FK-506

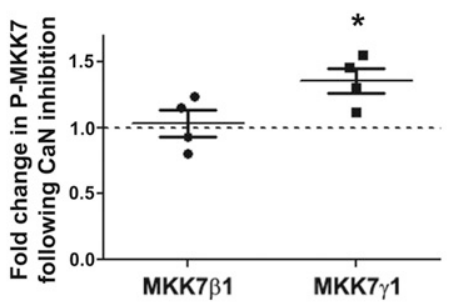

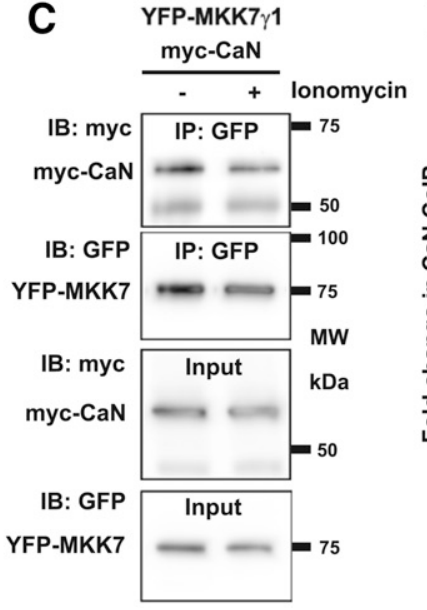

D

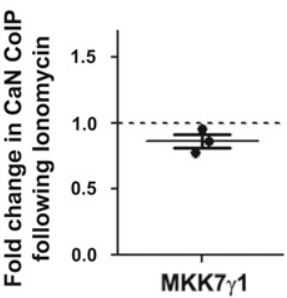

Fig. 8. CaN selectively regulates MKK7 $\gamma$ phosphorylation state. (A) YFP-MKK7 $\gamma 1$ or $\beta 1$ underwent IP from HEK-293FT cell lysates, and samples were immunoblotted (IB) with anti-P-MKK7 to detect phosphorylation at residues Ser271 and Thr275 or anti-GFP to detect total YFP-MKK7 expression. Coexpression of myc-CaN with YFP-MKK7 was confirmed by immunoblotting of the input cell lysates. (B) Quantification of four independent experiments, such as in (A), showing the fold change with CaN inhibition for P-MKK7 normalized to total YFP-MKK7 expression. Inhibition of CaN by CsA and FK-506 significantly increased phosphorylation of MKK7 $\gamma 1$ but not of MKK7 $\beta 1$ compared with untreated control conditions [fold change normalized to untreated controls MKK7 $\gamma 11.4 \pm 0.10$; MKK7 $\beta 1,1.0 \pm 0.1, n=4$; ${ }^{*} P<0.05$ for MKK7 $\gamma 1$ but $P>0.05$ (n.s.) for MKK7 $\beta 1$ to 1.0 by onesample $t$ test]. (C) Co-IP of myc-CaNA with YFP-MKK7 $\gamma 1$ after treatment with ionomycin or vehicle. (D) Quantification of co-IP of CaNA with MKK7 $\gamma 1$ revealed no significant impact of elevating intracellular calcium with ionomycin $(0.86 \pm 0.05 ; n=3$ independent experiments; one-sample $t$ test, $P>0.05)$. Please note, though not significant, the trend toward reduced interaction between CaNA and MKK7 $\gamma$ is likely related to a nonsignificant trend toward reduced total input levels of myc-CaNA $(0.67 \pm 0.11, P>0.05)$ in response to ionomycin in these same experiments.

either MKK7 $\gamma$ (Fig. 7C) or CaNA (Fig. 7D), and both proteins remained enriched in the cytoplasm relative to the nucleus, similar to control conditions, as indicated by the cytoplasm/nucleus ratio values, all of which were significantly $>1.0$. Furthermore, coexpression of a catalytically inactive mutant of CaN (CaNA-H151A-YFP) with CFP-MKK7 $\gamma$ (Fig. 7B) did not alter the cytoplasm versus nucleus distributions of MKK7 $\gamma$ or CaNA, and additional treatment with CsA also had no effect (Fig. 7, C and D). Thus, CaN-MKK7 $\gamma$ binding, rather than dephosphorylation, retains MKK7 $\gamma$ in the cytoplasm.

Nonetheless, having demonstrated a preferential association between $\mathrm{CaN}$ and $\mathrm{MKK} 7 \gamma$ relative to $\mathrm{MKK} 7 \beta$, we next asked whether $\mathrm{CaN}$ may be more effective at regulating the activity of MKK7 $\gamma$. MKK7 catalytic activity is governed by the phosphorylation of two residues in its kinase domain: Ser271 and Thr275. As such, an antibody to phospho-MKK7 (Ser271/Thr275) is a useful proxy for its activity level. We therefore expressed CaNA and either YFP-MKK7 $\beta 1$ or YFP-MKK71 $\gamma$ in HEK-293FT cells and treated them with vehicle or a CaN inhibitor cocktail $(2 \mu \mathrm{M}$ CsA, $2 \mu \mathrm{M}$ FK-506) for 30 minutes. IP of MKK7 followed by immunoblotting for P-MKK7 revealed a significant increase in P-MKK $7 \gamma 1$ signal in response to CaN inhibition (Fig. 8, A and B). However, no change in the P-MKK7 $\beta 1$ signal was detected. Overall, these findings highlight a role for $\mathrm{CaN}$ in the isoformspecific regulation of MKK7 activity that is conferred by the presence of a high-affinity PxIxIT motif in MKK7 $\gamma 1$. Having established isoform-specific regulation of MKK7 $\gamma$ phosphorylation by $\mathrm{CaN}$, we next asked whether $\mathrm{CaN}$ interaction with MKK7 $\gamma$ was regulated by intracellular calcium. We treated cells expressing myc-CaNA and YFP-MKK7 $\gamma$ with ionomycin $(1 \mu \mathrm{M}$, 3 minutes) and assessed the amount CaNA associated with MKK $7 \gamma$ by co-IP. Consistent with the known $\mathrm{Ca}^{2+}$-independent nature other CaNA-PxIxIT binding interactions (Li et al., 2011), no significant difference was detected in the amount of CaNA associated with MKK7 after ionomycin treatment (Fig. 8, C and D).
JNK signaling regulates important functions in the brain, including normal neuronal development and apoptotic neuronal cell death after excitotoxic insults (Davies and Tournier, 2012). Accordingly, inspection of the Allen Mouse Brain Atlas (Lein et al., 2007) for in situ hybridization data for MKK7 mRNA (Mapkk7 gene) using a probe that detects all six isoforms revealed that MKK7 mRNA is abundantly expressed in multiple brain regions (Fig. 9A), including the cerebellum, cortex, and hippocampus, with particularly high levels in the cerebellum and hippocampus (Fig. 9B). In the hippocampus, MKK7 mRNA expression is seen in cell bodies of pyramidal neurons in the CA1 and CA3 regions and of granule neurons in the dentate gyrus (Fig. 9, B and C). To assay for the expression of the MKK7 $\gamma$ isoform, we performed qRT-PCR with $\gamma$-specific primers on RNA isolated from mouse cerebellum, cortex, and hippocampus, and readily detected the expression of MKK7 $\gamma$ mRNA at similar relative levels to the housekeeping control gene GAPDH (Fig. 9D). However, when we performed qRT-PCR on RNA prepared from primary cultures of rat hippocampal neurons and cerebellar granule neurons, which also contain glial cells, we detected little or no MKK $7 \gamma$ mRNA expression (Fig. 9D). Closer inspection of in situ hybridization images revealed that MKK7 mRNA also appeared to be expressed in vascular structures in brain sections (Fig. 9C). We next performed qRT-PCR analysis of RNA from cultured rat microvascular endothelial cells and cultured rVSMCs, and detected MKK7 $\gamma$ mRNA expression only in rVMSCs (Fig. 9D). Thus, the detection of MKK7 $\gamma$ mRNA expression in RNA isolated from the brain is likely due to its expression in brain vascular smooth muscle cells (VSMCs).

To explore potential interactions between CaN and MKK7JNK signaling in VSMCs, we treated cultured rVSMCs with arginine-vasopressin (AVP), a vasoconstrictive/hypertrophic agonist of Gq-coupled V1a receptors that stimulate $\mathrm{Ca}^{2+}$ mobilization, muscle contraction, and smooth musclespecific gene expression through pathways that can involve both JNK and CaN signaling (Fig. 10A) (Higashita et al., 1997; 

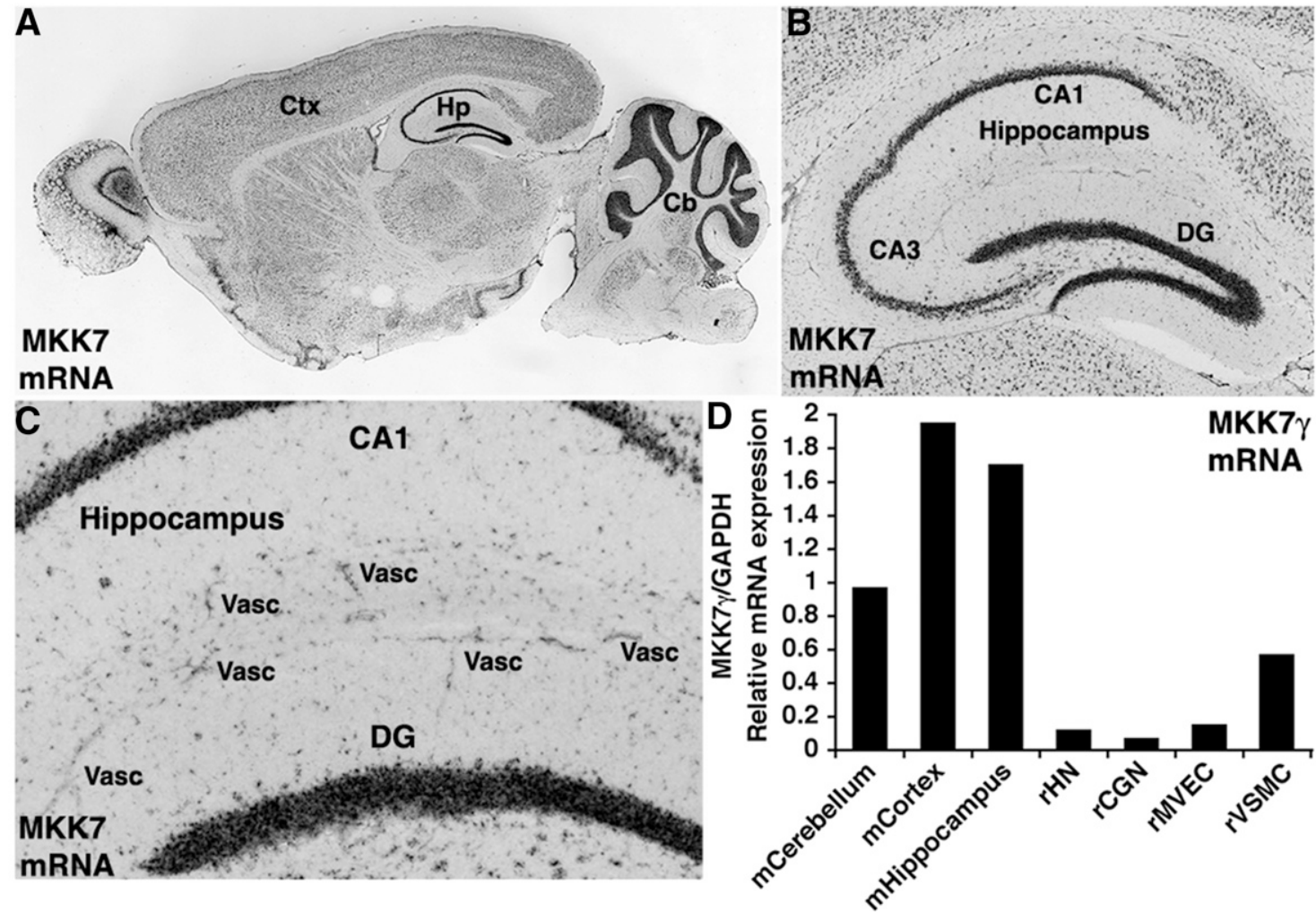

Fig. 9. MKK7 $\gamma$ mRNA is expressed in the brain and cultured VSMCs, but not in cultured neurons. (A) In situ hybridization detecting the expression of mRNA for all isoforms of MKK7 in a sagittal section of mouse brain. The cortex (Ctx), cerebellum (Cb), and hippocampus (Hp) are labeled. (B and C) Enlargements of the hippocampal region from (A) showing the expression of MKK7 mRNA in neuronal cell bodies of the CA1, CA3, and DG regions. Putative vascular structures (Vasc) expressing MKK7 mRNA in the hippocampus are labeled in (C). MKK7 mRNA in situ hybridization images in (A-C) downloaded from the Allen Mouse Brain Atlas (open access available from: http://mouse.brain-map.org). (D) Real-time PCR to quantitate MKK7 $\gamma$ mRNA expression relative to GAPDH mRNA expression in mouse $(\mathrm{m})$ cerebellum, cortex, and hippocampus; and in rat cultured hippocampal neurons (rHNs), cerebellar granule neurons (rCGNs), microvascular endothelial cells (rMVEC), and rVSMCs.

Nemenoff, 1998; Garat et al., 2000; Hill-Eubanks et al., 2003; Kaplan-Albuquerque et al., 2003b; Gonzalez Bosc et al., 2005). As expected, AVP treatment for 5 minutes increased P-JNK levels in a dose-dependent manner compared with untreated control cells (Fig. 10B). Importantly, incubation of rVSMCs with the CaN phosphatase inhibitor CsA further increased AVP-stimulated P-JNK levels (Fig. 10B), indicating negative regulation of the JNK pathway by $\mathrm{CaN}$. This effect of CsA on JNK signaling in rVSMCs was confirmed and quantified by JNK IP kinase assays using recombinant glutathione $S$ transferase GST-c-jun as a substrate (Fig. 10C). We observed that CsA + AVP led to a significant increase in JNK activity above those that produced by AVP alone (Fig. 10C). Overall, these data demonstrate cross talk between CaN and MKK7JNK signaling in rVSMCs, where CaN acts as a negative regulator of the JNK pathway and is consistent with the physical and functional interaction between $\mathrm{MKK} 7 \gamma$ and $\mathrm{CaN}$ identified above in HEK-293 and COS7 cells.

\section{Discussion}

Using a combination of in silico bioinformatics, in vitro biochemical analysis, and FRET imaging in live cells, we successfully characterized a novel binding interaction between the $\gamma$ isoform of the kinase MKK7 and the phosphatase CaN. In contrast, we found that the MKK7 $\alpha$ and $\beta$ isoforms do not directly interact with $\mathrm{CaN}$, although the closely related $\beta$ isoform may interact indirectly with $\mathrm{CaN}$ through other proteins. Highaffinity binding between $\mathrm{CaN}$ and MKK7 $\gamma$ in vitro depended on a consensus PxIxIT-type docking motif (41PIIVIT46) that is present only in the $\gamma$ isoform because of alternative splicing of exon 2 within the $\mathrm{N}$-terminal regulatory domain. Importantly, this consensus PxIxIT motif was required for direct binding between $\mathrm{CaN}$ and MKK7 $\gamma$ detected in the cytoplasm of living cells by FRET microcopy and prevented MKK7 $\gamma$ from localizing more prominently in the nucleus, like the MKK $7 \alpha$ and $\beta$ isoforms. Importantly, we also demonstrated that phosphorylation of MKK7 $\gamma$, but not of MKK7 $\beta$, is regulated by CaN phosphatase activity. Finally, we found that MKK7 $\gamma$ was expressed in VSMCs where it may promote negative cross-regulation of JNK signaling by $\mathrm{CaN}$ in response to stimulation of $\mathrm{G}$ protein-coupled receptor signaling by vasoconstrictive/hypertrophic agonists.

Based on peptide competition binding data, the strong consensus PIIVIT motif in MKK7 $\gamma$ binds CaN nearly as well as PVIVIT and AKAP79 IAIIIT, thus making it one of the highest-affinity known CaN binding partners. In contrast, the PTLQLP motif in MKK7 $\beta$ displays little or no direct $\mathrm{CaN}$ binding, thus the observed co-IP of $\mathrm{CaN}$ and $\mathrm{MKK} 7 \beta$ must be stabilized by indirect interactions with other proteins associated through the MKK7 N-terminal domain that is not present in MKK $7 \alpha$. In particular, the MKK7 N-terminal domain contains multiple JNK binding determinants including two LxL sites, 30LNL32 located just $\mathrm{N}$ terminal to and 59LQL61 located just $\mathrm{C}$ terminal to the strong consensus 
A

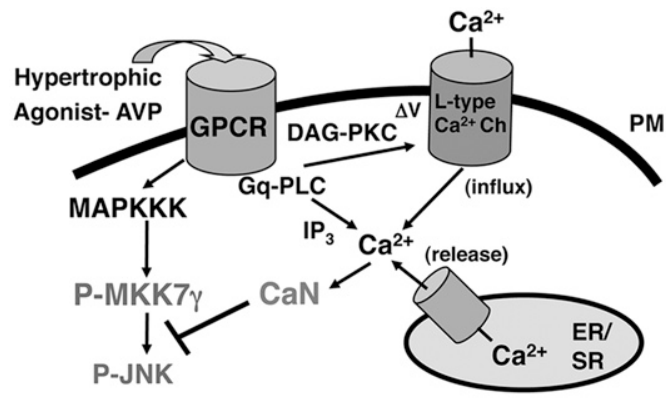

B

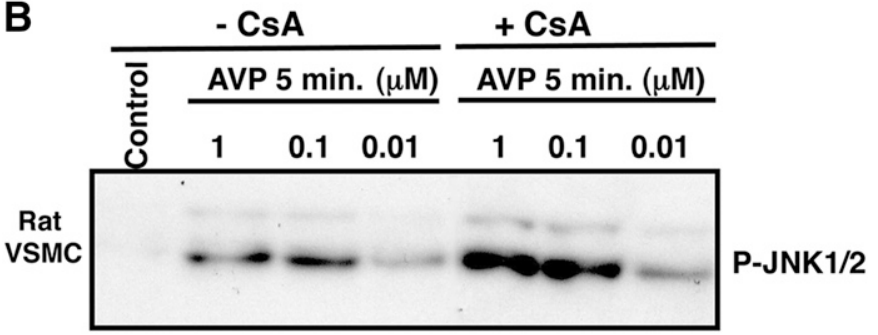

C

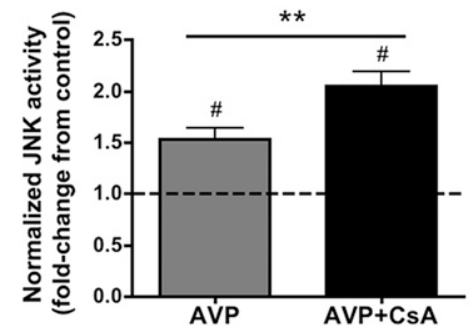

Fig. 10. CaN phosphatase activity inhibits JNK signaling in rVSMCs. (A) Diagram of potential negative regulation of MKK7-JNK signaling downstream of G protein-coupled receptor (GPCR) activation by AVP. (B) Incubation with $\mathrm{CsA}(0.8 \mu \mathrm{M})$ increases JNK1/2 phosphorylation (P-JNK1/2) in rVSMCs in response to increasing doses of AVP $(0.01-1 \mu \mathrm{M}, 5$ minutes $)$ as indicated. Phospho-JNK was detected by immunoblotting with an antibody recognizing the dual-Thr/Tyr phosphorylated JNK1/2 activation loop. (C) $\mathrm{CsA}(0.8 \mu \mathrm{M})$ increases $(0.1 \mu \mathrm{M}) \mathrm{AVP}$-stimulated JNK activity (normalized to untreated control cells). JNK activity was measured in cell extracts of rVSMCs using an in vitro IP-kinase assay that monitors ${ }^{32} \mathrm{P}$ transfer to recombinant GST-c-jun (in counts per minute) from $\left[\gamma_{-}{ }^{32} \mathrm{P}\right]$-ATP. Foldchange normalized to untreated controls: AVP, $1.53 \pm 0.10 ; \mathrm{AVP}+\mathrm{CsA}, 2.04$ $\pm 0.15 ; n=3 ; * * P=0.0075$ by paired $t$ test, which is below the Bonferronicorrected $P$ value threshold of $0.05 / 2=0.025$ for multiple comparisons; $\# P<$ 0.05 by one-sample $t$ test to 1.0 indicates significant AVP stimulation of JNK activity above respective untreated control conditions for both AVP alone and AVP+CsA.

PIIVIT site in MKK7 $\gamma$ (Tournier et al., 1999). In MKK7 $\beta$, this second LxL site is actually part of the nonconsensus 41PTLQLP46 site. Thus, JNK binding to the MKK7 $\beta / \gamma$ $\mathrm{N}$-terminal domain may link MKK7 and CaN to larger JNK signaling scaffolds, where $\mathrm{CaN}$ could interact with upstream MAPKKKs like ASK1, which can bind to the CaNB subunit (Liu et al., 2006), and/or scaffolds like JIP1 (Morrison and Davis, 2003). Alternatively, the close proximity of these JNK binding LxL sites to PIIVIT site in MKK7 $\gamma$ also raises the possibility of competition between CaN and JNK binding to MKK $7 \gamma$ N-terminal domain. For instance, binding of the antiapoptotic regulatory protein cellular FLICE-inhibitory protein to the $\mathrm{MKK} \beta / \gamma \mathrm{N}$ terminus competes with JNK binding (Nakajima et al., 2006). Thus, whether $\mathrm{CaN}$ competes with JNK binding and/or engages in interactions with additional binding partners in complexes with MKK7 $\beta$ and/or MKK7 $\gamma$ remains to be investigated.
Interestingly, the second LxL JNK binding motif in MKK7 $\gamma$ and $\beta$ is in the sequence context of LxLP, which is similar to a secondary CaN binding LxVP motif found in CaN substrates such as NFAT, the viral CaN inhibitory protein $\mathrm{A} 238 \mathrm{~L}$, and the PKA-RII regulatory subunit (Martinez-Martinez et al., 2006; Li et al., 2011; Grigoriu et al., 2013). LxVP motifs bind CaNA, not at the PxIxIT motif- $\beta$ sheet binding site in the catalytic domain, but at a site that is overlapping with where CaN-inhibitory immunosuppressant drug-immunophilin complexes (i.e., CsA-cyclophilin) bind in the hinge between the CaNA catalytic domain and C-terminal regulatory domain, where CaNB binds (Rodriguez et al., 2009; Grigoriu et al., 2013). Thus, MKK7 $\gamma$ could potentially use both a consensus PxIxIT motif and an LxVP-related motif to interact with CaNA, whereas MKK7 $\beta$ could potentially very weakly bind to CaN using either PTLQLP as a variant of a PxIxIT motif or LQLP as a variant of the LxVP motif. Consistent with this possibility, $\mathrm{CaN}$ was recently shown to bind to an LxL JNK binding motif in the downstream JNK target c-Jun (Goldman et al., 2014). Such an interaction of the MKK7 $\beta$ LQLP motif at the CaN LxVP binding site could also explain the sensitivity of the MKK7 $\beta$-CaN interaction to competition by PVIVIT, despite a lack of FRET in cells or of high-affinity direct binding in vitro, as it is known that at high concentrations PxIxIT motif peptides can cross-bind to the LxVP site (Martinez-Martinez et al., 2006). However, although we found that retention of MKK7 $\gamma$ in the cytoplasm by $\mathrm{CaN}$ required its binding to the PxIxIT motif, inhibition of $\mathrm{CaN}$ substrate binding at the LxVP binding site with the immunosuppressant CsA did not alter MKK7 $\gamma$ localization. Thus, although our results cannot completely rule out secondary CaNA binding to an LxVP-like motif in MKK7 $\gamma$ or $\beta$, they indicate that this site is not essential for interaction between MKK7 $\gamma$ and CaN.

Our data support a model in which CaN binding to MKK7 $\gamma$ in the cytoplasm allows $\mathrm{CaN}$ to regulate MKK7-JNK signaling preferentially in this compartment, as opposed to the nucleus, where $M K K 7 \alpha$ and $\beta$ may play more important roles. Although the catalytic activity of $\mathrm{CaN}$ was not required for maintaining CaNA and MKK7 $\gamma$ in the cytoplasm, CaN was capable of regulating MKK7 $\gamma$ activity through dephosphorylation of the Ser and Thr residues in the MKK7 activation loop. Alternatively, CaN scaffolding to MKK7 $\gamma$ may also position it for dephosphorylation of upstream MAPKKKs on Ser/Thr or downstream JNKs on Thr in their activation loops. Interestingly, MKK7 is a more specific regulator of JNK than MKK4, which also has some weak activity toward p38. In addition, MKK7 preferentially phosphorylates JNK on Thr in the activation loop compared with MKK4, which phosphorylates Thr and Tyr more equally (Wang et al., 2007). Importantly, JNKs are unique within the MAPK superfamily in that they can be substantially regulated by Thr phosphorylation alone. Thus, dephosphorylation of either MKK7 Ser/Thr phosphorylation or JNK Thr phosphorylation by $\mathrm{CaN}$ anchored to MKK7 $\gamma$ would be effective in inhibiting the JNK pathway activity as we observed in rVSMCs. However, future studies will need to be undertaken to specifically address the role for $\mathrm{CaN}$ anchoring to MKK7 $\gamma$ and its PxIxIT motif in these different possible mechanisms of JNK regulation in rVSMCs. Future studies will also need to be undertaken to specifically address whether cross talk between CaN and MKK7-JNK is signaling is preferentially directed to the cytoplasm, where the CaN-MKK7 $\gamma$ complex is found in addition to previously 
described cross talk in the nucleus, where CaN binds to c-Jun (Goldman et al., 2014).

Regardless of the detailed mechanisms, such cross talk between $\mathrm{CaN}$ and JNK could be very important for the regulation of vascular remodeling during injury and progression of atherosclerosis where there is important balanced regulation of VSMC differentiation by growth factors, like platelet-derived growth factor, and vasoconstrictive agonists, like AVP, between contractile/smooth muscle and motile/proliferative fibroblastlike phenotypes (Nemenoff, 1998; Kaplan-Albuquerque et al., 2003a,b). Importantly, vasoconstrictive agonists, like AVP and UTP, activate smooth muscle-specific gene expression programs associated with the contractile phenotype through both JNK regulation of the transcription factor serum response factor and CaN activation of NFAT (Nemenoff, 1998; Garat et al., 2000; Hill-Eubanks et al., 2003; Gonzalez Bosc et al., 2005). Thus, CaN binding to MKK7 $\gamma$ in the cytoplasm could negatively regulate JNK signaling to serum response factor in the nucleus both by promoting MKK7 retention in the cytoplasm and by inhibiting JNK activation/phosphorylation. However, JNK itself can act as a negative regulator of CaN-NFAT signaling in vascular smooth muscle by phosphorylating NFAT4/c3 in the nucleus to promote export (Gomez et al., 2003). Thus, CaN negative regulation of MKK7-JNK signaling may also have positive effects on CaN-NFAT activation in VSMCs. Needless to stay, numerous exciting possibilities for cross-regulation of CaN and MKK7-JNK signaling in VSMCs remain to be explored that could be important for understanding the mechanisms of vascular injury/repair both normally and during vascular disease states.

\section{Authorship Contributions}

Participated in research design: Gibson, Woolfrey, Li, Hogan, Nemenoff, Heasley, Dell'Acqua.

Conducted experiments: Gibson, Woolfrey, Li, Heasley.

Performed data analysis: Gibson, Woolfrey, Li, Hogan, Heasley, Dell'Acqua.

Wrote or contributed to the writing of the manuscript: Gibson, Woolfrey, Li, Hogan, Nemenoff, Heasley, Dell'Acqua.

\section{References}

Aramburu J, Garcia-Cozar F, Raghavan A, Okamura H, Rao A, and Hogan PG (1998) Selective inhibition of NFAT activation by a peptide spanning the calcineurin targeting site of NFAT. Mol Cell 1:627-637.

Aramburu J, Yaffe MB, Lopez-Rodriguez C, Cantley LC, Hogan PG, and Rao A (1999) Affinity-driven peptide selection of an NFAT inhibitor more selective than cyclosporin A. Science 285:2129-2133.

Carr DW, Hausken ZE, Fraser ID, Stofko-Hahn RE, and Scott JD (1992) Association of the type II cAMP-dependent protein kinase with a human thyroid RII-anchoring protein. Cloning and characterization of the RII-binding domain. J Biol Chem $\mathbf{2 6 7}$ 13376-13382.

Davies C and Tournier C (2012) Exploring the function of the JNK (c-Jun N-terminal kinase) signalling pathway in physiological and pathological processes to design novel therapeutic strategies. Biochem Soc Trans 40:85-89.

Dell'Acqua ML, Dodge KL, Tavalin SJ, and Scott JD (2002) Mapping the protein phosphatase-2B anchoring site on AKAP79. Binding and inhibition of phosphatase activity are mediated by residues 315-360. J Biol Chem 277:48796-48802.

Erickson MG, Liang H, Mori MX, and Yue DT (2003) FRET two-hybrid mapping reveals function and location of L-type $\mathrm{Ca} 2+$ channel CaM preassociation. Neuron 39:97-107.

Garat C, Van Putten V, Refaat ZA, Dessev C, Han SY, and Nemenoff RA (2000) Induction of smooth muscle alpha-actin in vascular smooth muscle cells by arginine vasopressin is mediated by c-Jun amino-terminal kinases and p38 mitogenactivated protein kinase. J Biol Chem 275:22537-22543.

Goldman A, Roy J, Bodenmiller B, Wanka S, Landry CR, Aebersold R, and Cyert MS (2014) The calcineurin signaling network evolves via conserved kinase-phosphatase modules that transcend substrate identity. Mol Cell $\mathbf{5 5}$ $422-435$

Gomez MF, Bosc LV, Stevenson AS, Wilkerson MK, Hill-Eubanks DC, and Nelson MT (2003) Constitutively elevated nuclear export activity opposes Ca2+-dependent NFATc3 nuclear accumulation in vascular smooth muscle: role of JNK2 and Crm-1. J Biol Chem 278:46847-46853.
Gonzalez Bosc LV, Layne JJ, Nelson MT, and Hill-Eubanks DC (2005) Nuclear factor of activated $\mathrm{T}$ cells and serum response factor cooperatively regulate the activity of an alpha-actin intronic enhancer. J Biol Chem 280:26113-26120.

Gorski JA, Gomez LL, Scott JD, and Dell'Acqua ML (2005) Association of an A-kinase-anchoring protein signaling scaffold with cadherin adhesion molecules in neurons and epithelial cells. Mol Biol Cell 16:3574-3590.

Grigoriu S, Bond R, Cossio P, Chen JA, Ly N, Hummer G, Page R, Cyert MS, and Peti W (2013) The molecular mechanism of substrate engagement and immunosuppressant inhibition of calcineurin. PLoS Biol 11:e1001492.

Higashita R, Li L, Van Putten V, Yamamura Y, Zarinetchi F, Heasley L, and Nemenoff RA (1997) Galpha16 mimics vasoconstrictor action to induce smooth muscle alpha-actin in vascular smooth muscle cells through a Jun-NH2-terminal kinase-dependent pathway. J Biol Chem 272:25845-25850.

Hill-Eubanks DC, Gomez MF, Stevenson AS, and Nelson MT (2003) NFAT regulation in smooth muscle. Trends Cardiovasc Med 13:56-62.

Kaplan-Albuquerque N, Garat C, Desseva C, Jones PL, and Nemenoff RA (2003a) Platelet-derived growth factor-BB-mediated activation of Akt suppresses smooth muscle-specific gene expression through inhibition of mitogen-activated protein kinase and redistribution of serum response factor. J Biol Chem 278:39830-39838.

Kaplan-Albuquerque N, Garat C, Van Putten V, and Nemenoff RA (2003b) Regulation of SM22 alpha expression by arginine vasopressin and PDGF-BB in vascular smooth muscle cells. Am J Physiol Heart Circ Physiol 285:H1444-H1452.

Lein ES, Hawrylycz MJ, Ao N, Ayres M, Bensinger A, Bernard A, Boe AF, Boguski MS, Brockway KS, Byrnes EJ, et al. (2007) Genome-wide atlas of gene expression in the adult mouse brain. Nature 445:168-176.

Li H, Pink MD, Murphy JG, Stein A, Dell'acqua ML, and Hogan PG (2012) Balanced interactions of calcineurin with AKAP79 regulate $\mathrm{Ca}(2+)$-calcineurin-NFAT signaling. Nat Struct Mol Biol 19:337-345.

Li H, Rao A, and Hogan PG (2004) Structural delineation of the calcineurin-NFAT interaction and its parallels to PP1 targeting interactions. J Mol Biol 342:1659-1674

$\mathrm{Li} \mathrm{H}$, Rao A, and Hogan PG (2011) Interaction of calcineurin with substrates and targeting proteins. Trends Cell Biol 21:91-103.

Li H, Zhang L, Rao A, Harrison SC, and Hogan PG (2007) Structure of calcineurin in complex with PVIVIT peptide: portrait of a low-affinity signalling interaction. $J$ Mol Biol 369:1296-1306.

Liu Q, Wilkins BJ, Lee YJ, Ichijo H, and Molkentin JD (2006) Direct interaction and reciprocal regulation between ASK1 and calcineurin-NFAT control cardiomyocyte death and growth. Mol Cell Biol 26:3785-3797.

Martinez-Martinez S, Rodriguez A, Lopez-Maderuelo MD, Ortega-Perez I, Vazquez J, and Redondo JM (2006) Blockade of NFAT activation by the second calcineurin binding site. J Biol Chem 281:6227-6235.

Morrison DK and Davis RJ (2003) Regulation of MAP kinase signaling modules by scaffold proteins in mammals. Annu Rev Cell Dev Biol 19:91-118.

Murphy JG, Sanderson JL, Gorski JA, Scott JD, Catterall WA, Sather WA and Dell'Acqua ML (2014) AKAP-anchored PKA maintains neuronal L-type calcium channel activity and NFAT transcriptional signaling. Cell Rep 7:1577-1588.

Nakajima A, Komazawa-Sakon S, Takekawa M, Sasazuki T, Yeh WC, Yagita H, Okumura K, and Nakano H (2006) An antiapoptotic protein, c-FLIPL, directly binds to MKK7 and inhibits the JNK pathway. EMBO J 25:5549-5559.

Nemenoff RA (1998) Vasopressin signaling pathways in vascular smooth muscle. Front Biosci 3:d194-d207.

Oliveria SF, Dell'Acqua ML, and Sather WA (2007) AKAP79/150 anchoring of calcineurin controls neuronal L-type $\mathrm{Ca} 2+$ channel activity and nuclear signaling. Neuron 55:261-275.

Oliveria SF, Dittmer PJ, Youn DH, Dell'acqua ML, and Sather WA (2012) Localized calcineurin confers $\mathrm{Ca} 2+$-dependent inactivation on neuronal L-type $\mathrm{Ca} 2+$ channels. $J$ Neurosci 32:15328-15337.

Oliveria SF, Gomez LL, and Dell'Acqua ML (2003) Imaging kinase-AKAP79phosphatase scaffold complexes at the plasma membrane in living cells using FRET microscopy. J Cell Biol 160:101-112.

Rodriguez A, Roy J, Martinez-Martinez S, Lopez-Maderuelo MD, Nino-Moreno P, Orti L, Pantoja-Uceda D, Pineda-Lucena A, Cyert MS, and Redondo JM (2009) A conserved docking surface on calcineurin mediates interaction with substrates and immunosuppressants. Mol Cell 33:616-626.

Sanderson JL, Gorski JA, Gibson ES, Lam P, Freund RK, Chick WS, and Dell'Acqua ML (2012) AKAP150-anchored calcineurin regulates synaptic plasticity by limiting synaptic incorporation of Ca2+-permeable AMPA receptors. J Neurosci 32: 15036-15052.

Schnell U, Dijk F, Sjollema KA, and Giepmans BN (2012) Immunolabeling artifacts and the need for live-cell imaging. Nat Methods 9:152-158.

Sorkin A, McClure M, Huang F, and Carter R (2000) Interaction of EGF receptor and grb2 in living cells visualized by fluorescence resonance energy transfer (FRET) microscopy. Curr Biol 10:1395-1398.

Tournier C, Whitmarsh AJ, Cavanagh J, Barrett T, and Davis RJ (1999) The MKK7 gene encodes a group of c-Jun NH2-terminal kinase kinases. Mol Cell Biol 19:1569-1581.

Wang X, Destrument A, and Tournier C (2007) Physiological roles of MKK4 and MKK7: insights from animal models. Biochim Biophys Acta 1773:1349-1357.

Wild AR and Dell'Acqua ML (2018) Potential for therapeutic targeting of AKAP signaling complexes in nervous system disorders. Pharmacol Ther 185:99-121.

Woolfrey KM and Dell'Acqua ML (2015) Coordination of protein phosphorylation and dephosphorylation in synaptic plasticity. J Biol Chem 290:28604-28612.

Woolfrey KM, Sanderson JL, and Dell'Acqua ML (2015) The palmitoyl acyltransferase DHHC2 regulates recycling endosome exocytosis and synaptic potentiation through palmitoylation of AKAP79/150. J Neurosci 35:442-456.

Address correspondence to: Mark L. Dell'Acqua, Department of Pharmacology, University of Colorado School of Medicine, Anschutz Medical Campus, 12800 East 19th Avenue, Mail Stop 8303, Aurora, CO 80045. E-mail: mark. dellacqua@ucdenver.edu 